\title{
A numerically efficient parametrization of turbulent wind-turbine flows for different thermal stratifications
}

4 Antonia Englberger · Andreas Dörnbrack

6 Received: DD Month YEAR / Accepted: DD Month YEAR

\begin{abstract}
The wake characteristics of a wind turbine in a turbulent atmospheric boundary layer under different thermal stratifications are investigated by means of large-eddy simulation with the geophysical flow solver EULAG. The turbulent inflow is based on a method that imposes the spectral energy distribution of a neutral boundary-layer precursor simulation, the turbulence preserving method. This method is extended herein to make it applicable for different thermal stratification regimes (convective, stable, neutral) by including suitable turbulence assumptions, which are deduced from velocity fields of a diurnal-cycle precursor simulation. The wind-turbine-wake characteristics derived from simulations that include the parametrization result in good agreement with diurnal-cycle-driven wind-turbine simulations. Furthermore, different levels of accuracy are tested in the parametrization assumptions, representing the thermal stratification. These range from three dimensional matrices of the precursor-simulation wind field to individual values. The resulting wake characteristics are similar, even for the simplest parametrization set-up, making the diurnal-cycle precursor simulation non-essential for the wind-turbine simulations. Therefore, the proposed parametrization results in a computationally fast, simple, and efficient tool for analyzing the effects of different thermal stratifications on wind-turbine wakes by means of large-eddy simulation.
\end{abstract}

Keywords Atmospheric boundary layer - Diurnal cycle · Large-eddy simulation · Turbulence · Wind-turbine wake

A. Englberger

Institut für Physik der Atmosphäre, DLR Oberpfaffenhofen

E-mail: antonia.englberger@dlr.de

A. Dörnbrack

Institut für Physik der Atmosphäre, DLR Oberpfaffenhofen

E-mail: andreas.doernbrack@dlr.de 


\section{Introduction}

A wind turbine in the atmospheric boundary layer (ABL) is exposed to turbulent flow that is strongly influenced by the different thermal stratifications occurring throughout a full diurnal cycle. The impact of ABL turbulence on the wake of a wind turbine is far-reaching. It affects the streamwise wake extension as well as the velocity deficit and the turbulence in the wake, which have a strong influence on power production, fatigue loading, and on the life expectancy of a wind turbine (e.g. Wharton and Lundquist, 2012; Vanderwende and Lundquist, 2012; Sathe et al., 2013; Dörenkämper et al., 2015).

Numerical simulations of diurnal-cycle-driven atmospheric flow through a wind turbine are necessary for a more detailed understanding of the windturbine-wake behaviour over the course of a day. This is essential as basis for increasing the power production of a wind turbine and to reduce the fatigue loading.

The influence of turbulence on the wake structure has been investigated in experimental (Medici and Alfredsson, 2006; Chamorro and Porté-Agel, 2009; Zhang et al., 2012) and numerical studies (Troldborg et al., 2007; Naughton et al., 2011; Wu and Porté-Agel, 2012; Witha et al., 2014; Englberger and Dörnbrack, 2017a,b): A more rapid wake recovery exists for incoming flow with higher turbulence intensity.

In numerical simulations there are various methods for generating and ensuring the turbulent flow field upstream of the wind turbine; in Mann (1994) a simple method was proposed, offering a synthetic turbulence field that is not based on a physical model (Naughton et al., 2011). This method has been applied in other studies, such as Troldborg et al. (2007). Mesoscale models apply nesting with one-way or two-way coupling to provide turbulent inflow in the microscale models (Mirocha et al., 2013; Muñoz-Esparza et al., 2014). An alternative approach is to use the data of a precursor simulation to simulate the flow around a wind turbine with periodic (Wu and Porté-Agel, 2012) or open (Naughton et al., 2011; Witha et al., 2014; Englberger and Dörnbrack, 2017a,b) boundary conditions in the streamwise direction. In each case, a large-eddy simulation (LES) is required that resolves the scales of turbulence relevant for the flow through the wind turbine. The generation of realistic turbulent inflow conditions is necessary to develop appropriate wind-farm set-ups that maximize power production and minimize fatigue loading on the turbines.

However, there are different numerical restrictions that have to be considered when applying a precursor LES in a wind-turbine simulation performed with open streamwise boundary conditions. The simulation of the flow around a wind turbine has to be fed continuously with turbulence data from the precursor simulation to ensure a fully-developed turbulent flow field. Continuous data input can be avoided by applying the turbulence preserving method as a parametrization of the turbulent inflow, as proposed by Englberger and Dörnbrack (2017a). This parametrization uses only one selected timestep from the precursor simulation when the flow is in equilibrium. In this way, the computational costs and the required memory for the simulation are greatly 
reduced. The turbulence preserving method from Englberger and Dörnbrack (2017a), however, is only valid for neutral stratification.

Buoyancy due to thermal stratification is one of the dominant mechanisms of production and suppression of atmospheric turbulence. The diurnal variation of the ABL is the result of external forcings such as varying surface heat fluxes, represented by heating during the day and cooling at night. These diurnal variations result in convection during the day and stable stratification at night (Stull, 1988), with the morning transition and the evening transition representing the corresponding transitional periods, that are defined following Grimsdell and Angevine (2002) as the time period in which the sensible heat flux changes sign. The morning ABL and the evening ABL include the atmospheric situation approximately half an hour before and after these transitions.

These different atmospheric stratifications affect the turbulence intensity, that in turn influences the wake structure. The response of the wind-turbine wake has been investigated in LES considering a stable ABL (Aitken et al., 2014; Abkar and Porté-Agel, 2014; Bhaganagar and Debnath, 2014, 2015; Abkar et al., 2016; Englberger and Dörnbrack, 2017b), a convective ABL (Mirocha et al., 2014; Abkar and Porté-Agel, 2014; Abkar et al., 2016; Englberger and Dörnbrack, 2017b), a neutral ABL (Abkar and Porté-Agel, 2014; Englberger and Dörnbrack, 2017a), the situation before and after the evening transition (Lee and Lundquist, 2017), as well as an evening ABL or a morning ABL (Englberger and Dörnbrack, 2017b). All investigations show a higher (weaker) turbulence intensity in the convective (stable) case, corresponding to a more (less) rapid wake recovery and a smaller (larger) velocity deficit during the day (night). The wake characteristics of the transitional periods (evening ABL, morning ABL) are mainly influenced by the respective flow regime (convective $\mathrm{ABL}$, stable $\mathrm{ABL}$ ) prior to the transition.

Therefore, numerical simulations of the complete diurnal cycle are required to produce realistic wake structures for the convective $\mathrm{ABL}$, the stable $\mathrm{ABL}$, the evening ABL, and the morning ABL. A diurnal-cycle simulation, representing all of these states, is published in Englberger and Dörnbrack (2017b). These diurnal-cycle simulations, however, are computationally very expensive and to minimize the computational costs we expand the turbulence preserving method from Englberger and Dörnbrack (2017a) towards a parametrization that is applicable for different atmospheric stratifications by combining it with the diurnal-cycle precursor simulation from Englberger and Dörnbrack (2017b). Furthermore, we test different levels of accuracy for the assumptions made by the parametrization to reduce the costs of the computationally very expensive precursor diurnal-cycle simulation.

The outline of the paper is as follows: the LES model, the wind-turbine parametrization, and the wind-turbine characteristics are presented in Sect. 2. The background wind profiles for different thermal stratifications are formulated in Sect. 3 and the parametrization of a stratification-dependent turbulent inflow condition in Sect. 4. The numerical experiments and the results follow in Sect. 5 and Sect. 6, while Conclusions are given in Sect. 7. Detailed derivations applied in Sect. 3 and Sect. 4 are given in the Appendices. 


\section{Numerical model framework}

\subsection{Numerical model EULAG}

The incompressible, turbulent, and dry flow through a wind turbine is simulated with the multiscale geophysical flow solver EULAG (Prusa et al., 2008; Englberger and Dörnbrack, 2017a), which refers to the ability of solving the equations of motions either in an EUlerian (flux form) (Smolarkiewicz and Margolin, 1993) or in a semi-LAGrangian (advective form) (Smolarkiewicz and Pudykiewicz, 1992) mode. The geophysical flow solver EULAG is at least second-order accurate in time and space (Smolarkiewicz and Margolin, 1998). Furthermore, it is well suited for massively-parallel computations (Prusa et al., 2008) and can be run in parallel up to a domain decomposition in three dimensions. A comprehensive description and discussion of the geophysical flow solver EULAG is given in Smolarkiewicz and Margolin (1998) and Prusa et al. (2008).

The Boussinesq equations are solved for the Cartesian velocity components $\mathbf{v}=(u, v, w)$ and for the potential temperature perturbations $\Theta^{\prime}=\Theta-\Theta_{e}($ Smolarkiewicz et al., 2007),

$$
\begin{aligned}
\frac{d \mathbf{v}}{d t} & =-G \nabla\left(\frac{p^{\prime}}{\rho_{0}}\right)+\mathbf{g} \frac{\Theta^{\prime}}{\Theta_{0}}+\mathcal{V}+\mathbf{M}+\frac{\mathbf{F}_{W T}}{\rho_{0}}, \\
\frac{d \Theta^{\prime}}{d t} & =\mathcal{H} \\
\nabla \cdot\left(\rho_{0} \mathbf{v}\right) & =0
\end{aligned}
$$

for a flow with constant density $\rho_{0}=1.1 \mathrm{~kg} \mathrm{~m}^{-3}$ and a constant reference value of the potential temperature $\Theta_{0}=301 \mathrm{~K}$. Height dependent states $\psi_{e}(\mathrm{z})=$ $\left(u_{e}(\mathrm{z}), v_{e}(\mathrm{z}), w_{e}(\mathrm{z}), \Theta_{e}(\mathrm{z})\right)$ enter Eqs. $1-3$ in the buoyancy term and as boundary conditions. These background states correspond to the ambient or environmental states. Initial conditions are provided for $u, v, w$, and the potential temperature perturbation $\Theta^{\prime}$. In the following, $\psi=\left(u, v, w, \Theta^{\prime}\right)$ shall denote the prognostic variables analyzed from the EULAG simulations in Sect. 5. In Eqs. 1,2 , and $3, d / d t, \nabla$, and $\boldsymbol{\nabla}$. represent the total derivative, the gradient and the divergence, respectively. The factor $G$ represents geometric terms resulting from the general, time-dependent coordinate transformation (Wedi and Smolarkiewicz, 2004; Smolarkiewicz and Prusa, 2005; Prusa et al., 2008; Kühnlein et al., 2012), $p^{\prime}$ represents the pressure perturbation with respect to the environmental state, and $\mathbf{g}$ is the vector of the acceleration due to gravity. The subgrid-scale terms $\mathcal{V}$ and $\mathcal{H}$ symbolise viscous dissipation of momentum and diffusion of heat and $\mathbf{M}$ denotes the inertial forces of coordinate-dependent metric accelerations. $\mathbf{F}_{W T}$ corresponds to the turbine-induced force, implemented with the blade element momentum method as rotating actuator disc in the simulations (Englberger and Dörnbrack, 2017a). All the following simu- 
lations are performed with a turbulent kinetic energy (TKE) closure (Schmidt and Schumann, 1989; Margolin et al., 1999).

In general, the geophysical flow solver EULAG owes its versatility to a unique design that combines a rigorous theoretical formulation in generalized curvilinear coordinates (Smolarkiewicz and Prusa, 2005) with non-oscillatory forward-in-time differencing for fluids built on the multi-dimensional positive definite advection transport algorithm, which is based on the convexity of upwind advection (Smolarkiewicz and Margolin, 1998; Prusa et al., 2008) and a robust, exact-projection type, elliptic Krylov solver (Prusa et al., 2008). The flow solver has been applied to a wide range of scales simulating various problems like turbulence (Smolarkiewicz and Prusa, 2002), flow past complex or moving boundaries (Wedi and Smolarkiewicz, 2006; Kühnlein et al., 2012), gravity waves (Smolarkiewicz and Dörnbrack, 2008; Doyle et al., 2011) or even solar convection (Smolarkiewicz and Charbonneau, 2013).

\subsection{Wind-turbine simulations}

Three different simulation types, investigating the flow around a wind turbine are performed in our study: benchmark, reference, and parametrization wind-turbine simulations. A detailed description follows in Sect. 5. All windturbine simulations are performed for different stratifications lasting $1 \mathrm{~h}$, with a horizontal resolution of $5 \mathrm{~m}$ and a vertical resolution of $5 \mathrm{~m}$ in the lowest $200 \mathrm{~m}$ and $10 \mathrm{~m}$ above. The benchmark wind-turbine simulations are performed on $512 \times 512 \times 64$ grid points with open streamwise and periodic spanwise boundary conditions. The reference and the parametrization windturbine simulations are performed on on $512 \times 64 \times 64$ grid points with open horizontal boundary conditions. In all wind-turbine simulations, no surface fluxes are applied. The rotor of the wind turbine is located at $300 \mathrm{~m}$ in $x$ - direction and centred in $y$ - direction of the corresponding domain with a diameter $D$ of $100 \mathrm{~m}$ and a hub height $z_{h}$ of $100 \mathrm{~m}$. The axial $\mathbf{F}_{x}$ and tangential $\mathbf{F}_{\Theta}$ turbine-induced forces $\left(\mathbf{F}_{W T}=\mathbf{F}_{x}+\mathbf{F}_{\Theta}\right)$ in Eq. 1 are parameterized with the blade element momentum method as rotating actuator disc with a nacelle, covering $20 \%$ of the blades. The forces account for different wind speeds and local blade characteristics and are parameterized with the airfoil data from the $10 \mathrm{MW}$ reference wind turbine from DTU (Technical University of Denmark) (Mark Zagar (Vestas), personal communication), whereas the radius of the rotor as well as the chord length of the blades are scaled to a rotor with a diameter of $100 \mathrm{~m}$. A detailed description of the wind-turbine parametrization and the applied smearing of the forces, as well as all values used in the wind-turbine parametrization are given in Englberger and Dörnbrack (2017a, parametrization B).

\subsection{Wind-turbine characteristics}

The wind-turbine wake is characterized by the following diagnostic quantities: 
- The spatial distribution of the streamwise velocity component $\overline{u_{i, j, k}}$, the streamwise velocity ratio

$$
V R_{i, j, k} \equiv \frac{\overline{u_{i, j, k}}}{\overline{u_{1, j, k}}},
$$

and the streamwise velocity deficit

$$
V D_{i, j, k} \equiv \frac{\overline{u_{1, j, k}}-\overline{u_{i, j, k}}}{\overline{u_{1, j, k}}}
$$

as they are related to the power loss of a wind turbine. The indices of the grid points are denoted by $i=1 \ldots n, j=1 \ldots m$, and $k=1 \ldots l$ in the $x$, $y$, and $z$ directions, respectively. The upstream velocity $u_{1, j, k}$ is taken at the first upstream grid point in the $x$-direction and the corresponding $y$ and $z$ coordinates.

- The total turbulent intensity

$$
I_{i, j, k}=\frac{\frac{1}{3} \sqrt{\sigma_{u_{i, j, k}}^{2}+\sigma_{v_{i, j, k}}^{2}+\sigma_{w_{i, j, k}}^{2}}}{\overline{u_{i, j, k_{h}}}},
$$

with $\sigma_{u_{i, j, k}}=\sqrt{\overline{\overline{u_{i, j, k}^{\prime 2}}}}, \sigma_{v_{i, j, k}}=\sqrt{\overline{v_{i, j, k}^{\prime 2}}}$, and $\sigma_{w_{i, j, k}}=\sqrt{\overline{\overline{w_{i, j, k}^{\prime 2}}}}$, as well as $u_{i, j, k}^{\prime}=u_{i, j, k}-\overline{u_{i, j, k}}, v_{i, j, k}^{\prime}=v_{i, j, k}-\overline{v_{i, j, k}}$, and $w_{i, j, k}^{\prime}=w_{i, j, k}-\overline{w_{i, j, k}}$, as it affects the flow-induced dynamic loads on downwind turbines.

We perform all wind-turbine simulations for $60 \mathrm{~min}$, a period long enough for the wake to reach an equilibrium state with statistical convergence of the results. The mean values are averaged over the last $50 \mathrm{~min}$. The temporal average $\overline{\Psi_{x, y, z}}$ of a quantity $\Psi$ for a time period $t$ is calculated online in the numerical model and updated at every timestep according to the method of Fröhlich (2006, Eq. 9.1). Further, in the $x-z$ plane, the indices $j_{0}$ corresponds to the centre of the domain in $y$-direction, whereas in the $x-y$ plane, $k_{h}$ corresponds to the hub height $z_{h}$.

Generally, the numerical simulation results are plotted in the following in dimensionless coordinates as a function of the rotor diameter $D$. The contour of the actuator in the cross-sections represents the transition to a wind-turbine force of zero. Furthermore, only a section of the complete computational domain is shown in most of the following plots.

\section{Background wind profiles for different thermal stratifications}

For the derivation of the background wind profiles from LES for different thermal stratifications, two different precursor simulations are needed, a neutral ABL and a diurnal-cycle simulation. We apply the neutral ABL simulation from Englberger and Dörnbrack (2017a) and the diurnal-cycle simulation from Englberger and Dörnbrack (2017b). An overview of the numerical set-ups and the wind-field characteristics of both simulations is given in Appendix 1. 
From these precursor simulations, two different background wind fields for $u_{e}$ and $v_{e}$ (dashed lines in Fig. 1) can be extracted for the proposed parametrization:

- A daytime representation

$$
u_{e}(z)=\frac{u_{*}}{\kappa} \ln \left(\frac{z}{z_{0}}\right)
$$

for the convective ABL and the evening ABL profiles with a friction velocity $u_{*}=0.35 \mathrm{~m} \mathrm{~s}^{-1}$, a von Kármán constant $\kappa=0.4$, a roughness length $z_{0}=0.001 \mathrm{~m}$, and the meridional background wind profile $v_{e}(z)=0$.

- A night-time representation of the zonal and meridional wind profiles for the stable ABL and the morning ABL can be expressed by

$$
\begin{aligned}
& u_{e}(z)=u_{g}\left(1-\exp \left(-\frac{z \sqrt{f / K_{0}}}{\sqrt{2}}\right) \cos \left(\frac{z \sqrt{f / K_{0}}}{\sqrt{2}}\right)\right), \\
& v_{e}(z)=u_{g} \exp \left(-\frac{z \sqrt{f / K_{0}}}{\sqrt{2}}\right) \sin \left(\frac{z \sqrt{f / K_{0}}}{\sqrt{2}}\right),
\end{aligned}
$$

for an initialization time $t=0$, a Coriolis parameter $f=1.0 \times 10^{-4} \mathrm{~s}^{-1}$, geostrophic wind components $u_{g}=10 \mathrm{~m} \mathrm{~s}^{-1}$, and $v_{g}=0$, and an eddy viscosity coefficient $K_{0}=0.06 \mathrm{~m}^{2} \mathrm{~s}^{-1}$ according to Shapiro and Fedorovich (2010, Eqs. 36 and 37).

The vertical background wind profiles $w_{e}$ are set to zero for both the daytime and night-time representations.

\section{Parametrization of a stratification-dependent turbulent inflow condition}

The general idea of the turbulence preserving method is to sustain the background turbulence in an LES with open horizontal boundary conditions of a flow through a wind turbine by a numerically simple parametrization of the imposed turbulent fluctuations. The original version of the turbulence preserving method (Englberger and Dörnbrack, 2017a) is only applicable under neutral atmospheric conditions. To extend it for different atmospheric stratifications, we propose a modification. A detailed description of the original version of the turbulence preserving method as well as the necessary modifications to make it applicable for different atmospheric stratifications are given in Appendix 2, in the following, we focus on the modified turbulence preserving method.

The modified version of the turbulence preserving method is described by:

$$
\left.\mathbf{u}_{p}^{*}\right|_{i=1, j, k} ^{\xi}=\alpha_{0} \boldsymbol{\alpha}_{i^{*}, j, k} \underbrace{\left(\left.\mathbf{u}_{p}\right|_{i^{*}, j, k}-\left.\frac{1}{n m} \sum_{i=1}^{n} \sum_{j=1}^{m} \mathbf{u}_{p}\right|_{i, j, k}\right)}_{I} .
$$



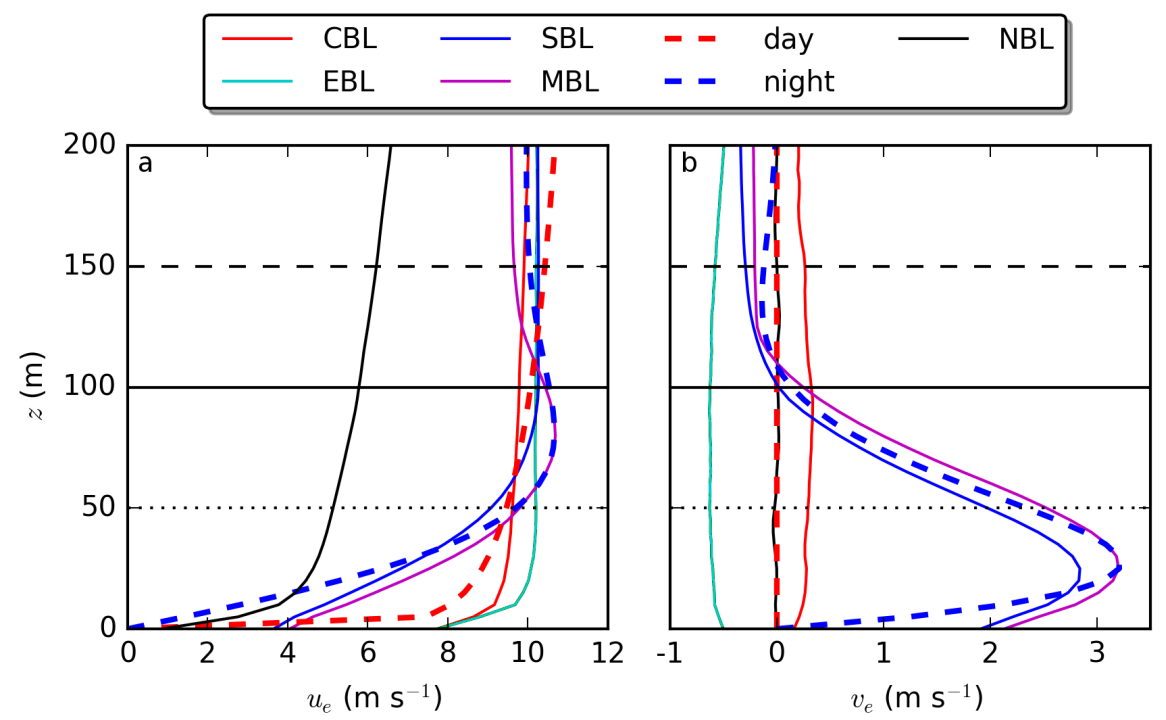

Fig. 1 Vertical profiles of the horizontal average of the background profiles $u_{e}$ in $(a)$ and $v e$ in $(b)$ for the neutral ABL (NBL), the convective ABL (CBL), the evening ABL (EBL), the stable ABL (SBL), and the morning ABL (MBL) precursor simulation. The applied fits for the day and the night are plotted as dashed lines. The hub height $(100 \mathrm{~m}$; black solid line), the top tip ( $150 \mathrm{~m}$; black dashed line) and the bottom tip (50 m; black dotted line) region of a wind turbine with $D=100 \mathrm{~m}$ and $z_{h}=100 \mathrm{~m}$ are highlighted by horizontal lines.

In Eq. 10, $\alpha_{0}$ is an adjustable value, and can be set to 1 in general applications of the parametrization. The stratification-dependent weighting parameters $\boldsymbol{\alpha}_{i^{*}, j, k}$ account for different atmospheric conditions and are explained in more detail in the following. The star refers to a streamwise direction shift by one grid point every timestep $\xi$, symbolized by $i^{*}=i+\xi^{*}$, with $i^{*} \in[1, n]$ and $\xi^{*}$ representing the number of timesteps since the start of the simulation. Further, $I$ represents the magnitude of the velocity perturbations. All other indices are explained in detail in Appendix 2.

The idea of this parametrization is to apply the wind field $\mathbf{u}_{p}$ of an equilibrium state of the neutral ABL precursor simulation in $I$ and include the turbulent flow characteristics of different stratifications via $\boldsymbol{\alpha}_{i^{*}, j, k}$. The most accurate representation of the turbulence structure would be the synchronized use of the three wind fields $u(x, y, z), v(x, y, z)$, and $w(x, y, z)$ of the diurnalcycle precursor simulation, a computationally extremely expensive approach. $u(x, y, z), v(x, y, z)$, and $w(x, y, z)$ are equivalent to the discrete representation $u_{i, j, k}, v_{i, j, k}$, and $w_{i, j, k}$ in the numerical model.

We therefore apply as first simplification three 3D matrices of the wind components $u, v$, and $w$ of the diurnal cycle at a certain time as function set $A$ for $\boldsymbol{\alpha}_{i^{*}, j, k}$. This, however, requires the knowledge of three wind fields of the neutral ABL precursor simulation and three wind fields for each atmospheric state of the diurnal-cycle precursor simulation, resulting in the necessity of 
performing the computational extremely expensive diurnal-cycle simulation as precursor simulation.

Therefore, we propose as further simplification and function set B the use of three vertical $1 \mathrm{D}$ vectors of $u, v$, and $w$, which are representative for the different atmospheric states. This is motivated by two reasons: Firstly, the vertical structure of $u, v$, and $w$ (Figs. 1 and 11) is much more distinct in comparison to the horizontal. Secondly, the effects of major changes for different situations (e.g. heterogeneous surface, more convective / stable situation (higher / lower surface heat flux in precursor simulation)) can be estimated from the three vertical profiles resulting from the diurnal cycle for each stratifications.

To completely eliminate the need of a detailed knowledge of $u, v$, and $w$ of a diurnal-cycle simulation, we propose as extreme simplification and function set $C$ the use of only a scalar for $u, v$, and $w$, respectively, for each atmospheric stratification. The values should refer to a height that is covered by the blades of the wind turbine. Therefore, this function set only requires the three wind fields of the neutral ABL precursor simulation for $I$, which makes it to a simple, numerically efficient, and computationally fast LES approach to represent wind-turbine wakes.

For the stratification-dependent weighting parameter $\boldsymbol{\alpha}_{i^{*}, j, k}$, the derivation of the three applied function sets representing different levels of accuracy are explained in detail in the flowing.

A: $\boldsymbol{\alpha}_{i^{*}, j, k}$ corresponds to three 3D matrices for each wind component, resulting in:

$$
\boldsymbol{\alpha}_{i^{*}, j, k}=\frac{1}{3}\left(\mathbf{u}_{\alpha_{i^{*}}}+\mathbf{u}_{\alpha_{j}}+\mathbf{u}_{\alpha_{k}}\right),
$$

defined as

$$
\begin{aligned}
& \mathbf{u}_{\alpha_{i}^{*}} \equiv \frac{\max \left(\mathbf{u}_{d c_{i, 1: m, 1: l}}\right)-\min \left(\mathbf{u}_{d c_{i, 1: m, 1: l}}\right)}{\max \left(\mathbf{u}_{N B L_{i, 1: m, 1: l}}\right)-\min \left(\mathbf{u}_{N B L_{i, 1: m, 1: l}}\right)}, \\
& \mathbf{u}_{\alpha_{j}} \equiv \frac{\max \left(\mathbf{u}_{d c_{1: n, j, 1: l}}\right)-\min \left(\mathbf{u}_{d c_{1: n, j, 1: l}}\right)}{\max \left(\mathbf{u}_{N B L_{1: n, j, 1: l}}\right)-\min \left(\mathbf{u}_{N B L_{1: n, j, 1: l}}\right)}, \\
& \mathbf{u}_{\alpha_{k}} \equiv \frac{\max \left(\mathbf{u}_{d c_{1: n, 1: m, k}}\right)-\min \left(\mathbf{u}_{d c_{1: n, 1: m, k}}\right)}{\max \left(\mathbf{u}_{N B L_{1: n, 1: m, k}}\right)-\min \left(\mathbf{u}_{N B L_{1: n, 1: m, k}}\right)} .
\end{aligned}
$$

Here, $\mathbf{u}_{d c_{i, j, k}}$ are the corresponding $3 \mathrm{D}$ wind fields of the convective ABL, the evening $\mathrm{ABL}$, the stable $\mathrm{ABL}$, or the morning $\mathrm{ABL}$, extracted from the diurnal-cycle precursor simulation, whereas $\mathbf{u}_{N B L_{i, j, k}}$ corresponds to the equilibrium state of the neutral ABL precursor simulation. The differences in the numerator as well as in the denominator correspond to the maximum of the fluctuations occurring in the corresponding atmospheric state. The maximum of the fluctuations of the neutral ABL state is used as suitable normalization. This is motivated by the term $I$ in Eq. 10, which is also derived from the neutral ABL simulation. Therefore, the three $1 \mathrm{D}$ vectors in Eqs. 12 - 14 include the spatial structure and the normalized turbulence. 
B: $\boldsymbol{\alpha}_{i^{*}, j, k}$ corresponds to three vertical 1D vectors for the three wind components, resulting in:

$$
\boldsymbol{\alpha}_{i^{*}, j, k}=\mathbf{u}_{\alpha_{k}}
$$

C: One value for all three wind components, valid at all grid points, resulting in $\alpha_{u}, \alpha_{v}$, and $\alpha_{w}$. The values are an approximation of the values of $\mathbf{u}_{\alpha_{k}}(z)$ taken at $100 \mathrm{~m}$, which corresponds to the hub height of a common wind turbine.

The vertical profiles of $\boldsymbol{\alpha}_{i^{*}, j, k}$ for different thermal stratifications for function set type $B$ are shown in Fig. 2 together with the horizontally averaged fluctuations of $u, v$, and $w$ for the neutral ABL, the convective ABL, the evening $\mathrm{ABL}$, the stable $\mathrm{ABL}$, and the morning ABL, respectively. The fluctuations contribute to the nominator as well as to the denominator of Eq. 14 . They are very small for the stable $\mathrm{ABL}$ and the morning $\mathrm{ABL}$, increase in the evening ABL, and are largest in the convective ABL. More precisely, they increase by a factor of eight from the stable ABL and the morning ABL to the evening ABL and by a factor of three from the evening ABL to the convective ABL. The magnitudes of neutral ABL fluctuations correspond most closely to the evening ABL values for all three wind components. Therefore, the values of $u_{\alpha_{k}}, v_{\alpha_{k}}$, and $w_{\alpha_{k}}$ in Fig. 2 are roughly one in the evening ABL, representing the transitional state. During the day (night), they are larger ( smaller) in comparison to the transitional state, resulting from a larger (smaller) numerator in comparison to the denominator in Eq. 14. Further, the values $\alpha_{u}, \alpha_{v}$, and $\alpha_{w}$ of function set type $C$ result from $u_{\alpha_{k}}, v_{\alpha_{k}}$, and $w_{\alpha_{k}}$ of function set type $B$ at hub height of $100 \mathrm{~m}$. They are listed in Table 1 for all three regimes.

Due to this same-sized fluctuations and stratification-dependent weighting values of function set type $B$ in the stable $\mathrm{ABL}$ and in the morning $\mathrm{ABL}$ all wind-turbine-simulation characteristics are also rather similar for the stable $\mathrm{ABL}$ and the morning $\mathrm{ABL}$ and are, therefore, only discussed for the stable ABL case in the following.

Therefore, we classify the following wind-turbine simulations herein into three regimes with two different background wind fields (Table 1):

- A daytime state, which is prescribed by a logarithmic background wind profile (Eq. 7) with relatively large wind fluctuations.

- A night-time state, which is prescribed by a background wind profile with a wind direction change with height (Eqs. 8 and 9) and very small wind fluctuations.

- A transition between the daytime and the night-time states, which is prescribed by the same logarithmic background wind profile (Eq. 7) as the daytime situation, however, characterized by smaller (larger) wind fluctuations in comparison to the day (night).

The parametrization proposed herein is tested in the following wind-turbine simulations for these three regimes. 

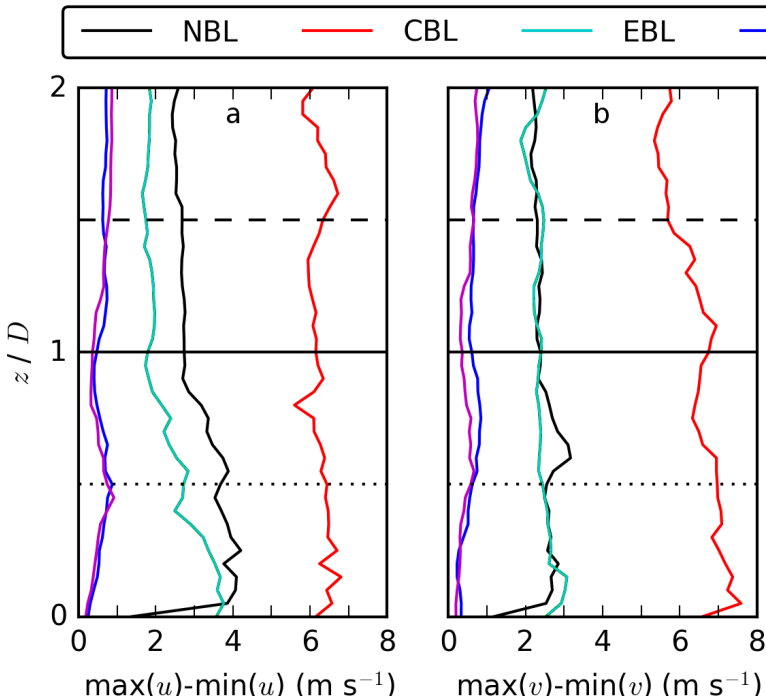

SBL $\longrightarrow$ MBL
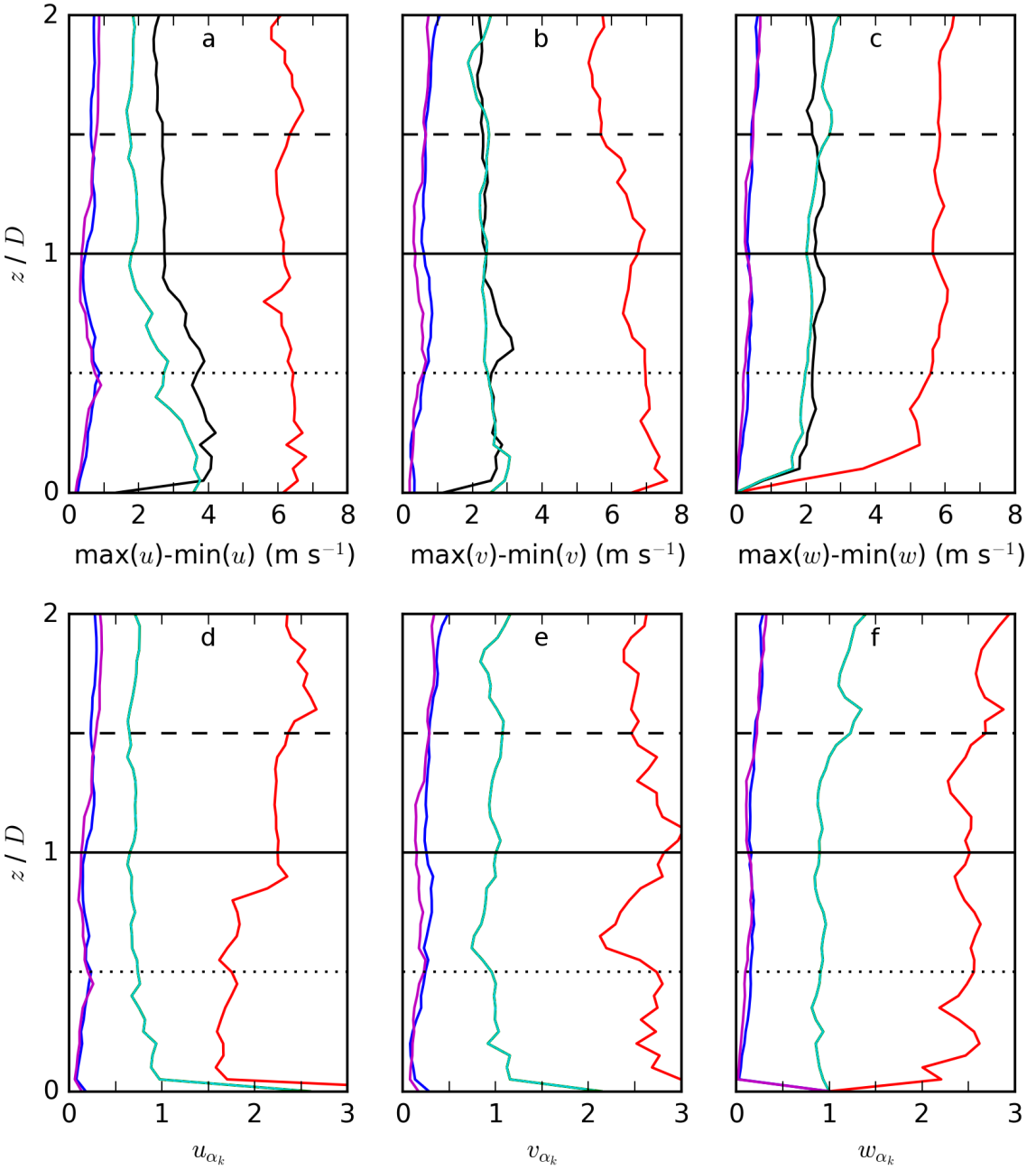

Fig. 2 Vertical profiles of the maximum of the difference of $u$ in $(a), v$ in $(b)$, and $w$ in (c), calculated as $\max \left(\mathbf{u}_{N B L_{1: n, 1: m, k}}\right)-\min \left(\mathbf{u}_{N B L_{1: n, 1: m, k}}\right)$ for the neutral ABL (NBL) and $\max \left(\mathbf{u}_{d c_{1: n, 1: m, k}}\right)-\min \left(\mathbf{u}_{d c_{1: n, 1: m, k}}\right)$ for the convective ABL (CBL), the evening ABL (EBL), the stable ABL (SBL), and the morning ABL (MBL) situations, respectively. Vertical profiles of $\boldsymbol{\alpha}_{i^{*}, j, k}$ are presented for $u_{\alpha_{k}}$ in $(d), v_{\alpha_{k}}$ in $(e)$, and $w_{\alpha_{k}}$ in $(f)$ of type $B$ calculated with Eq. 14 for the convective ABL, the evening ABL, the stable ABL, and the morning ABL situation. 
Table 1 List of the applied background wind profiles $u_{e}, v_{e}$, and $w_{e}$ as well as the values $\alpha_{u}$, $\alpha_{v}$, and $\alpha_{w}$, used for the wind-turbine simulations representing the daytime, the transition, and the night-time situation.

\begin{tabular}{c|c|c|c} 
state & day & transition & night \\
\hline$u_{e}(z)$ & $\frac{u_{*}}{\kappa} \ln \left(\frac{z}{z_{0}}\right)$ & $\frac{u_{*}}{\kappa} \ln \left(\frac{z}{z_{0}}\right)$ & $u_{g}\left(1-\exp \left(-\frac{z \sqrt{f / K_{0}}}{\sqrt{2}}\right) \cos \left(\frac{z \sqrt{f / K_{0}}}{\sqrt{2}}\right)\right)$ \\
\hline$v_{e}(z)$ & 0 & 0 & $u_{g} \exp \left(-\frac{z \sqrt{f / K_{0}}}{\sqrt{2}}\right) \sin \left(\frac{z \sqrt{f / K_{0}}}{\sqrt{2}}\right)$ \\
\hline$w_{e}(z)$ & 0 & 0 & 0 \\
\hline$\alpha_{u}$ & 2.5 & 0.6 & 0.15 \\
\hline$\alpha_{v}$ & 2.8 & 1.0 & 0.13 \\
\hline$\alpha_{w}$ & 2.5 & 0.9 & \\
\hline
\end{tabular}

\section{Numerical Experiments}

Three different types of wind-turbine simulations are investigated, the benchmark, the reference, and the parametrization wind-turbine simulations. The schematic illustration in Fig. 3 shows their dependencies on the input conditions provided by the neutral ABL and the diurnal-cycle precursor simulations and the applied parametrization.

A benchmark wind-turbine simulation (Fig. 3, green) applies the background conditions $\psi_{e}=\left(u_{e}, v_{e}, w_{e}, \Theta_{e}\right)$ of the corresponding diurnal-cycle state and $2 \mathrm{D}$ slices of the temporal evolution of $\psi\left(\psi_{j, k}(t)\right)$ at each timestep.

Contrary to the benchmark wind-turbine simulations, the reference and the parametrization wind-turbine simulations consider the temporal fluctuations only in the wind field. No potential temperature deviations from the prescribed background profile $\Theta_{e}(z)=300 \mathrm{~K}$ are considered during the 1-h wind-turbine simulations, resulting in $\tilde{\psi}=(u, v, w)$ and $\tilde{\psi}_{e}=\left(u_{e}, v_{e}, w_{e}\right)$ instead of $\psi=\left(u, v, w, \Theta^{\prime}\right)$ and $\psi_{e}=\left(u_{e}, v_{e}, w_{e}, \Theta_{e}\right)$.

A reference wind-turbine simulation (Fig. 3, red) applies a simplified version of our proposed parametrization. The wind profiles $\tilde{\psi}_{d c}$ of the corresponding state of the diurnal cycle are the basis for the daytime and the night-time fits of the background wind profiles $\tilde{\psi}_{e}$ (Fig. 1, dashed lines). Further, the stratification dependent weighting parameter $\boldsymbol{\alpha}_{i^{*}, j, k}$ in Eq. 10 is set to 1 and the turbulent fluctuations of $u, v$, and $w$ in $I$ (Eq. 10) are extracted directly from the corresponding diurnal-cycle state, resulting in $2 \mathrm{D}$ slices of $\tilde{\psi}\left(\tilde{\psi}_{j, k}(t)\right)$. Together with the background wind profiles $\tilde{\psi}_{e}$, the parametrization can be applied, driving the reference wind-turbine simulations.

A parametrization wind-turbine simulation (Fig. 3, blue) applies the same background wind profiles in the parametrization as in the reference windturbine simulation. However, the wind profiles $\tilde{\psi}$ of the diurnal-cycle precursor simulation $\left(\tilde{\psi}_{d c}\right)$ as well as of the neutral ABL precursor simulation $\left(\tilde{\psi}_{N B L}\right)$ are used for the calculation of the stratification dependent weighting parameter $\boldsymbol{\alpha}_{i^{*}, j, k}$. Further, the wind profiles of the neutral ABL precursor simulations $\tilde{\psi}_{N B L}$ are applied in the calculation of the turbulent fluctuations of $u, v$, and $w$ in $I$ in Eq. 10. The arising $2 \mathrm{D}$ slices of $\psi\left(\tilde{\psi}_{j, k}(t)\right)$ in combination 


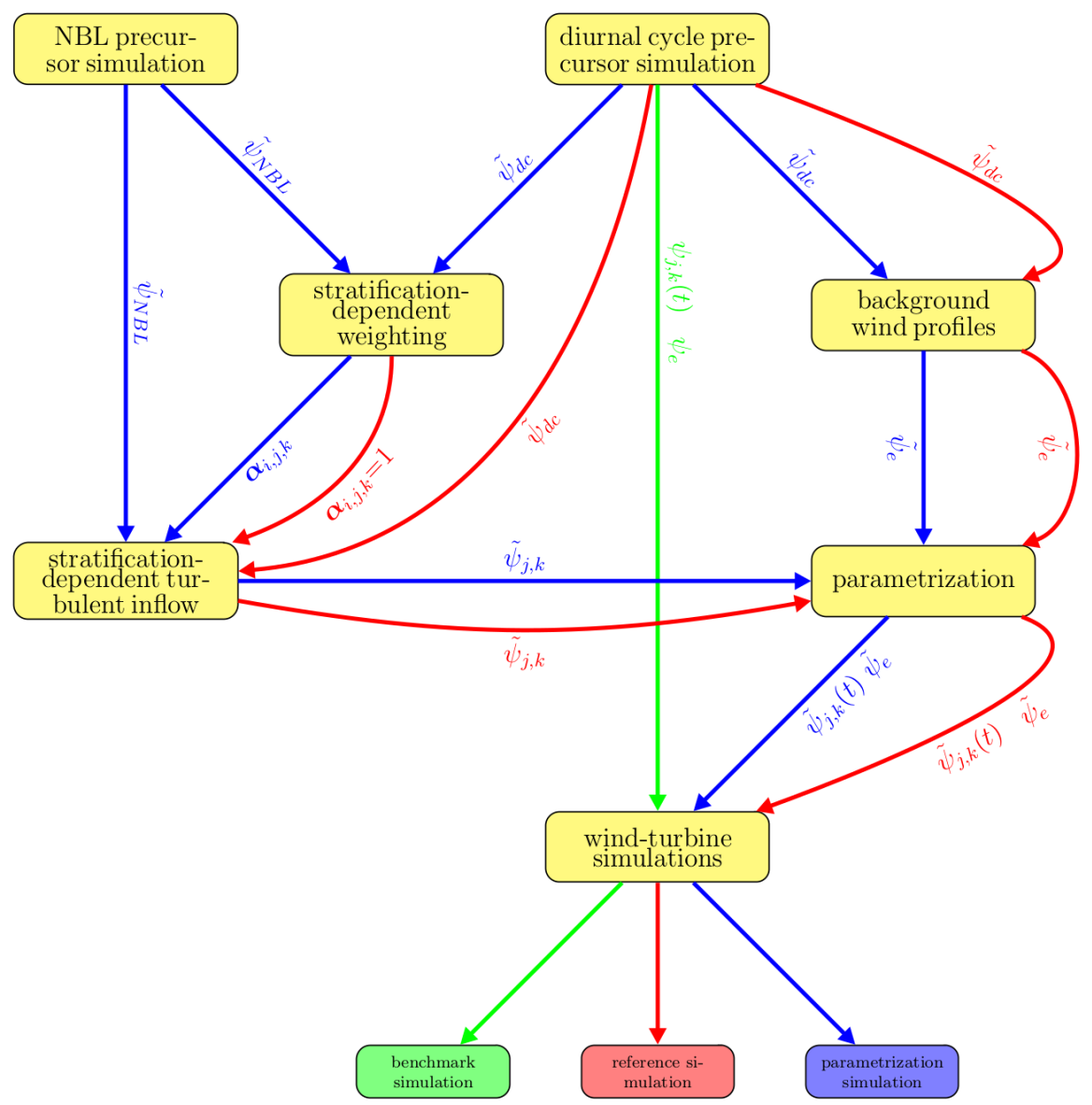

Fig. 3 Schematic of benchmark, reference, and parametrization wind-turbine simulations input, with $\psi=\left(u, v, w, \Theta^{\prime}\right), \psi_{e}=\left(u_{e}, v_{e}, w_{e}, \Theta_{e}\right), \tilde{\psi}=(u, v, w)$, and $\tilde{\psi}_{e}=\left(u_{e}, v_{e}, w_{e}\right)$.

with the background wind profiles $\tilde{\psi}_{e}$ allow the application of the proposed parametrization in the parametrization wind-turbine simulations.

The main characteristics of these three types of wind-turbine simulations are summarized in Table 2 and a more detailed description is given in the following:

\subsection{Benchmark wind-turbine simulations}

The benchmark wind-turbine simulations correspond to the synchronized diurnalcycle wind-turbine simulations over homogeneous surface in Englberger and Dörnbrack (2017b). The convective ABL, the evening ABL, and the stable ABL wind-turbine simulations are used as representatives for the daytime, 
Table 2 Summary of the main characteristics of the performed wind-turbine simulations. The subscript $I$ corresponds to Eq. 10 and CBL (EBL, SBL, NBL) to the convective (evening, stable, neutral) ABL.

\begin{tabular}{c|c|c|c} 
wind-turbine simulation type & benchmark & reference & parametrization \\
\hline parametrization & no & yes & yes \\
\hline precursor simulation for $I$ & CBL, EBL, SBL & CBL, EBL, SBL & NBL \\
\hline $\begin{array}{c}\text { coupling } \\
\text { method }\end{array}$ & synchronized & $12 \mathrm{~h}, 18 \mathrm{~h}, 24 \mathrm{~h}$ & equilibrium \\
diurnal-cycle state & diurnal-cycle state & NBL state \\
\hline
\end{tabular}

the transition, and the night-time regime. For the synchronized coupling between the diurnal-cycle precursor simulation and the wind-turbine simulations, the background fields $\psi_{e}(t)=\left(u_{e}(t), v_{e}(t), w_{e}(t), \Theta_{e}(t)\right)$ with $u_{e}(t)=<u(t)>_{z}$, $v_{e}(t)=<\quad v(t)>_{z}$, $w_{e}(t)=<w(t)>_{z}$, and $\Theta_{e}(t)=\Theta_{e}(t=0 \mathrm{~h})+<\Theta^{\prime}(t)>_{z}$, the initial fields, and the inflow data of all prognostic variables $\psi(t)=\left(u(t), v(t), w(t), \Theta^{\prime}(t)\right)$ are taken from the idealized diurnal-cycle precursor simulation after $t=12 \mathrm{~h}$ for the convective ABL, $t=18 \mathrm{~h}$ for the evening acABL, and $t=24 \mathrm{~h}$ for the stable ABL regime. Here, $t=12 \mathrm{~h}, 18 \mathrm{~h}$, and $24 \mathrm{~h}$ can be considered as 1200 , 1800,2400 local time. The horizontal averages of the initial conditions are taken as background profiles, as denoted by \langle\rangle$_{z}$. Due to the open streamwise boundary condition, the wind-turbine simulation has to be fed continuously with inflow data from the idealized diurnal-cycle precursor simulation. The inflow data are taken as 2D $y-z$ slices $\psi_{j, k}(t)$ at $i=\mathrm{n}$ of $\psi$ from the diurnal-cycle simulation at each timestep for 1-h time intervals from $t=12 \mathrm{~h}$ to $13 \mathrm{~h}$ for the convective $\mathrm{ABL}$, from $t=18 \mathrm{~h}$ to $19 \mathrm{~h}$ for the evening $\mathrm{ABL}$, and from $t=24 \mathrm{~h}$ to $25 \mathrm{~h}$ for the stable ABL, to ensure synchronized wind-turbine simulations. In the corresponding synchronized timestep of the benchmark wind-turbine simulation, $\psi_{j, k}(t)$ represents the upstream values of $\psi$ at $i=1$. This approach is described in more detail in Englberger and Dörnbrack (2017b).

Only the spanwise position $j$ of the diurnal-cycle precursor simulation $\left(\psi(t)_{j_{1} \leq j \leq j_{2}}\right)$, which interacts with the wind turbine, differs in the benchmark wind-turbine simulations performed in the scope of this paper $\left(j_{1}=22 ; j_{2}=42\right)$ from those in Englberger and Dörnbrack (2017b, Figs. 3 and 4$)\left(j_{1}=246\right.$; $\left.j_{2}=266\right)$. This difference is related to the use of another lateral sector $j_{1} \leq j \leq$ $j_{2}$ of the diurnal-cycle precursor simulation, which is applied in the corresponding wind-turbine simulation. Both benchmark wind-turbine simulations (here and in Englberger and Dörnbrack (2017b)) are performed on 512 spanwise grid points with periodic spanwise boundary conditions. In the wind-turbine simulations of Englberger and Dörnbrack (2017b), the wind turbine is located in the lateral domain centre $(m=512)$, whereas in the benchmark wind-turbine simulations presented herein, the wind turbine is located at $j=32$, corresponding to the domain centre in the following parametrization and reference windturbine simulations, which are performed on 64 grid points in spanwise direction with open spanwise boundary conditions. The resulting deviations of the 
wake deflection and the entrainment rate between these two types of benchmark wind-turbine simulations are negligibly small.

\subsection{Parametrization wind-turbine simulations}

The wind-turbine simulations performed with the proposed parametrization for the daytime, the transitional, and the night-time situation, are referred to hereafter as parametrization wind-turbine simulations of type $A, B$, and $C$. They correspond to the three sets of functions $A, B$, and $C$ for $\boldsymbol{\alpha}_{i^{*}, j, k}$ (Eq. 11).

A fit to the horizontal average of the atmospheric state of the wind in the diurnal-cycle precursor simulation $\tilde{\psi}(t)=(u(t), v(t), w(t))$ is applied for the background wind profiles $\tilde{\psi}_{e}(t)=\left(u_{e}(t), v_{e}(t), w_{e}(t)\right)$, as explained in Sect. 3 . The convective ABL regime at $t=12 \mathrm{~h}$ and the evening ABL regime at $t=18 \mathrm{~h}$ are approximated by a logarithmic zonal background wind profile (Eq. 7) and no meridional and vertical background wind. For the stable ABL, the atmospheric state after $t=24 \mathrm{~h}$ is considered to be best described by a wind direction change with height of the horizontal background wind profiles (Eqs. 8 and 9 ). All applied wind profiles that contribute to the parametrization, are listed in Table 1 . In addition to the background wind profiles, $\Theta_{e}(z)=300 \mathrm{~K}$ at all times and in all heights. In contrast to the benchmark wind-turbine simulations, no inflow data of the prognostic variables $\psi(t)=\left(u(t), v(t), w(t), \Theta^{\prime}(t)\right)$ are applied as 2D $y-z$ slices at each timestep, instead, $\tilde{\psi}_{i=1, j, k}(t)=\left.\mathbf{u}_{p}^{*}\right|_{i=1, j, k} ^{\xi}$ is modified by the use of the modified turbulence preserving method following Eq. 10, as explained in Sect. 4. This implies a consideration of the fluctuations only in the wind field, no potential temperature deviations from $\Theta_{e}$ are considered during the $1 \mathrm{~h}$ wind-turbine simulation.

Further, the stratification-dependent weightings $\boldsymbol{\alpha}_{i^{*}, j, k}$ are calculated by the use of $\tilde{\psi}_{N B L}$ of the neutral ABL precursor simulation and $\tilde{\psi}_{d c}$ of the corresponding regimes of the diurnal-cycle precursor simulation as three $3 \mathrm{D}$ matrices for parametrization type $A$, three $1 \mathrm{D}$ vectors for parametrization type $B$, or three values for parametrization type $C$. Here again, the $t=12 \mathrm{~h}$ $(18 \mathrm{~h}, 24 \mathrm{~h})$ regime is used for the daytime (transition, night-time) situation in Eqs. $12-14$.

The resulting stratification-dependent weightings $\boldsymbol{\alpha}_{i^{*}, j, k}$ and the wind conditions $\tilde{\psi}_{N B L}$ of the neutral ABL equilibrium state contribute to the calculation of the stratification-dependent turbulent inflow in Eq. 10 with $\alpha_{0}=0.5$. The value of $\alpha_{0}$ will be motivated later on.

\subsection{Reference wind-turbine simulation}

The parametrization wind-turbine simulations combine $\tilde{\psi}_{d c}$ and $\tilde{\psi}_{N B L}$ for the calculation of the stratification-dependent weightings $\boldsymbol{\alpha}_{i^{*}, j, k}$ and further apply $\tilde{\psi}_{N B L}$ in the modified version of the turbulence preserving method in Eq. 10. The contribution of $\tilde{\psi}_{N B L}$ in combination with $\boldsymbol{\alpha}_{i^{*}, j, k}$ is the general idea of our 
parametrization wind-turbine simulations. In contrast, the benchmark windturbine simulations consider $\psi_{j, k}(t)$ of the diurnal-cycle precursor simulation without applying the parametrization. Therefore, to examine the applicability of $\tilde{\psi}_{N B L}$ and $\boldsymbol{\alpha}_{i^{*}, j, k}$ in the parametrization wind-turbine simulations, there is a need for an additional type of simulations, the reference wind-turbine simulations.

The reference wind-turbine simulations apply the parametrization as shown in Fig. 3. Instead of including the neutral ABL precursor simulation in the calculation of the stratification-dependent weightings $\boldsymbol{\alpha}_{i^{*}, j, k}$ and in the modified version of the turbulence preserving method in Eq. 10, the stratificationdependent inflow uses $\tilde{\psi}_{d c}$ in the turbulence preserving method together with a stratification-dependent weighting of 1 and $\alpha_{0}=0.5$. The value of $\alpha_{0}$ corresponds to the parametrization wind-turbine simulations.

These reference wind-turbine simulations serve as simplification of the benchmark wind-turbine simulations with the following limitations: They consider only one time of the diurnal-cycle precursor simulation $(t=12 \mathrm{~h}$ for the day, $t=18 \mathrm{~h}$ for the transition, $t=24 \mathrm{~h}$ for the night) instead of the $1 \mathrm{~h}$ evolution of the atmospheric state $(t=12 \mathrm{~h}-13 \mathrm{~h}$ for the day, $t=18 \mathrm{~h}-19 \mathrm{~h}$ for the transition, $t=24 \mathrm{~h}-25 \mathrm{~h}$ for the night). Further, they do not include $\Theta^{\prime}$, resulting in $\tilde{\psi}(t)$ instead of $\psi(t)$. In addition, the background wind profiles are not the horizontal averages of the diurnal-cycle precursor simulation. Instead, the approximated profiles from Table 1 and Fig. 1 are applied. The $\Theta_{e}$ profile is constant with height at $300 \mathrm{~K}$. Further, the reference wind-turbine simulations are more complex in comparison to the parametrization wind-turbine simulations, as they directly consider a specific time of the 3D $u, v$, and $w$ fields of the precursor simulation, which is the most accurate representation of the turbulence structure, as explained in detail in Sect. 4 . Therefore, they require the diurnal-cycle precursor simulation. Considering these modifications, the reference wind-turbine simulations represent an intermediate step between the benchmark wind-turbine simulations and the parametrization wind-turbine simulations.

\subsection{Additional remarks on the simulations}

In the following, two specific remarks on the numerical experiments are given:

- The parametrization and the reference wind-turbine simulations, both performed with the stratification-dependent turbulent inflow from Eq. 10, are implemented with $\alpha_{0}=0.5$, as stated before. This value results in a slightly less (more) rapid wake recovery of the reference wind-turbine simulation in comparison to the benchmark wind-turbine simulation of the convective ABL (stable ABL). The best fit of the reference wind-turbine simulation with the benchmark wind-turbine simulation is achieved for $\alpha_{0}=0.7$ in the convective $\mathrm{ABL}$, for $\alpha_{0}=0.5$ in the evening $\mathrm{ABL}$, and for $\alpha_{0}=0.3$ in the stable ABL. Taking this into account, the following reference wind-turbine simulations and the parametrization wind-turbine simulations of type $A$, 
$B$, and $C$ are performed with $\alpha_{0}=0.5$, as this value results on average over all three regimes in the best fit of the wake structure of the reference wind-turbine simulation with the corresponding benchmark wind-turbine simulation.

- Daytime parametrization wind-turbine simulations of type $A, B$, and $C$ are further modified by applying the same perturbation velocities $\left.\mathbf{u}_{p}^{*}\right|_{i=1, j, k} ^{\xi}$ (Eq. 10) for three consecutive timesteps. This simple approach mimics the larger turbulent eddies prevalent in the convective ABL. The resulting simulated entrainment process results in a better agreement of parametrization wind-turbine simulations of type $A, B$, and $C$ with the corresponding reference wind-turbine simulations. This modification is only applied in the daytime parametrization wind-turbine simulations, not in the transitional and stable simulations.

\section{Results}

In the following, the three types of wind-turbine simulations (benchmark, reference, parametrization) are investigated in detail for the three ABL regimes (daytime, transition, nocturnal). The ultimate goal of the presented intercomparisons is to verify the applicability of the parametrization for different thermal stratifications.

The streamwise velocity component of the benchmark wind-turbine simulations (in a), the reference wind-turbine simulations (in b), and the parametrization wind-turbine simulations of type $A$ (in c), $B$ (in d), and $C$ (in e) are displayed in the $x-z$ plane in Figs. 4,6 , and 8 and in the $x-y$ plane in Figs. 5, 7, and 9. Figures 4 and 5 represent the daytime ABL regime, Figs. 6 and 7 the ABL of the transitional state, and Figs. 8 and 9 the nocturnal ABL regime.

The general wake structure of the simulated streamwise velocity component of all simulations in Figs. 4-9 reveals a deceleration of the flow right behind the rotor with a wind speed increase in radial and streamwise directions, resulting from the entrainment of air with higher velocity values from the surrounding air flow. The upstream region differs for the convective ABL and the evening $\mathrm{ABL}$ in comparison to the stable ABL and the morning ABL due to the distinct differences in the profiles of the zonal upstream velocity component in Fig. 1a. In the following, all wind-turbine simulations corresponding to the same ABL regime are compared.

\subsection{Benchmark and reference wind-turbine simulations}

As first step, the reference wind-turbine simulations are compared to the benchmark wind-turbine simulations to investigate the impact of the parametrization. Considering a stratification-dependent weighting $\boldsymbol{\alpha}_{i^{*}, j, k}$ of 1 in 

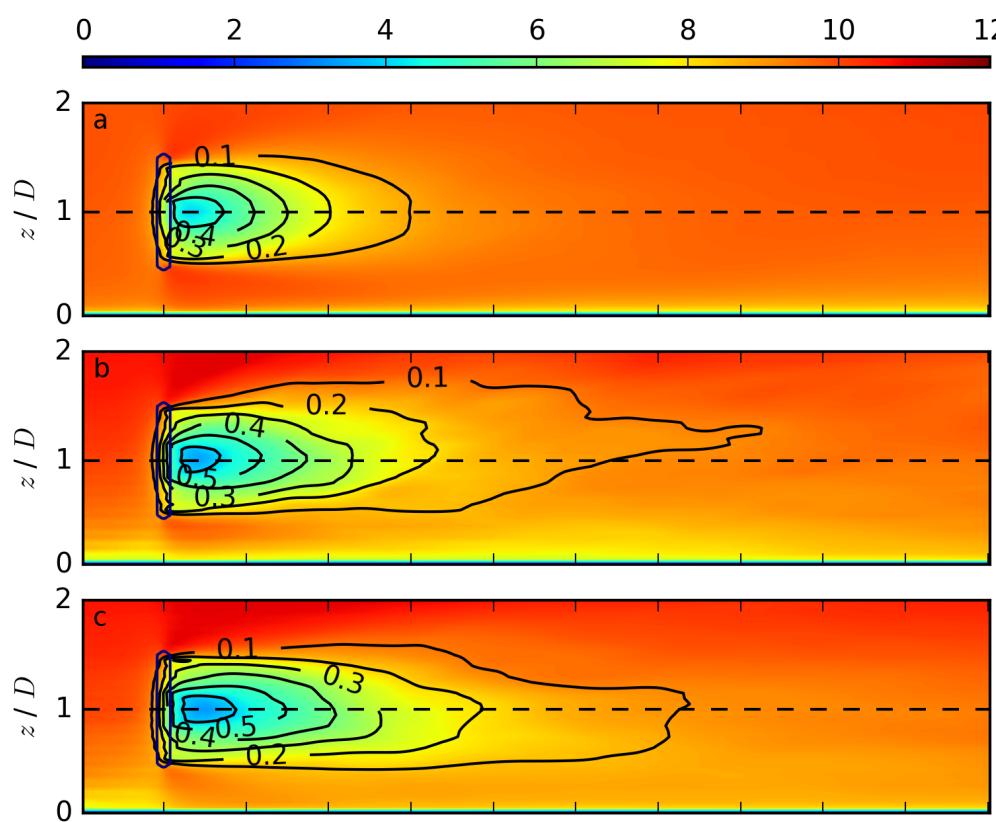

A

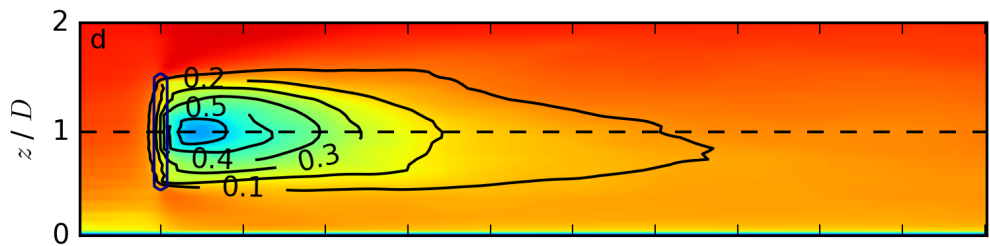

B



C

Fig. 4 Daytime vertical cross-sections of $\overline{u_{i, j_{0}, k}}$ in $\mathrm{m} \mathrm{s}^{-1}$ for the benchmark wind-turbine simulation in $(a)$, the reference wind-turbine simulation in $(b)$ and parametrization windturbine simulations of type $A, B$, and $C$ in $(c),(d)$, and $(e)$ respectively. The black contours represent $V D_{i, j_{0}, k}$. 


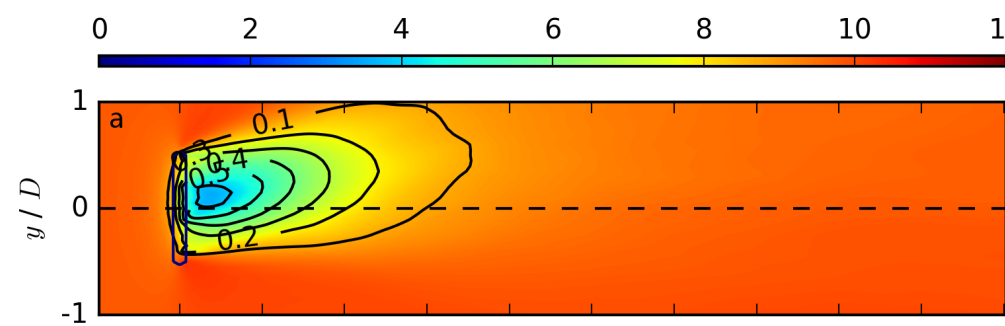

12


A

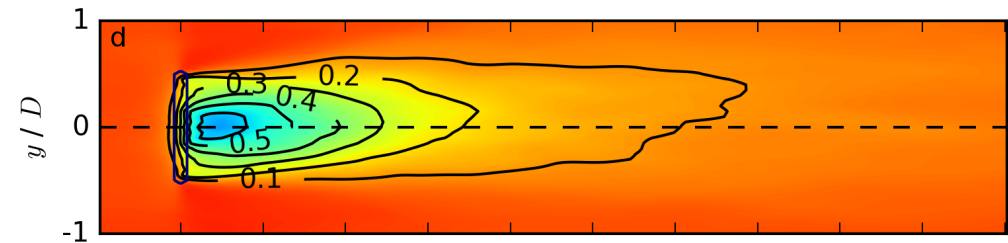

B



C

Fig. 5 Daytime horizontal cross-sections of $\overline{u_{i, j, k_{h}}}$ in $\mathrm{m} \mathrm{s}^{-1}$ for the benchmark wind-turbine simulation in $(a)$, the reference wind-turbine simulation in $(b)$ and parametrization windturbine simulations of type $A, B$, and $C$ in $(c),(d)$, and $(e)$ respectively. The black contours represent $V D_{i, j, k_{h}}$. 

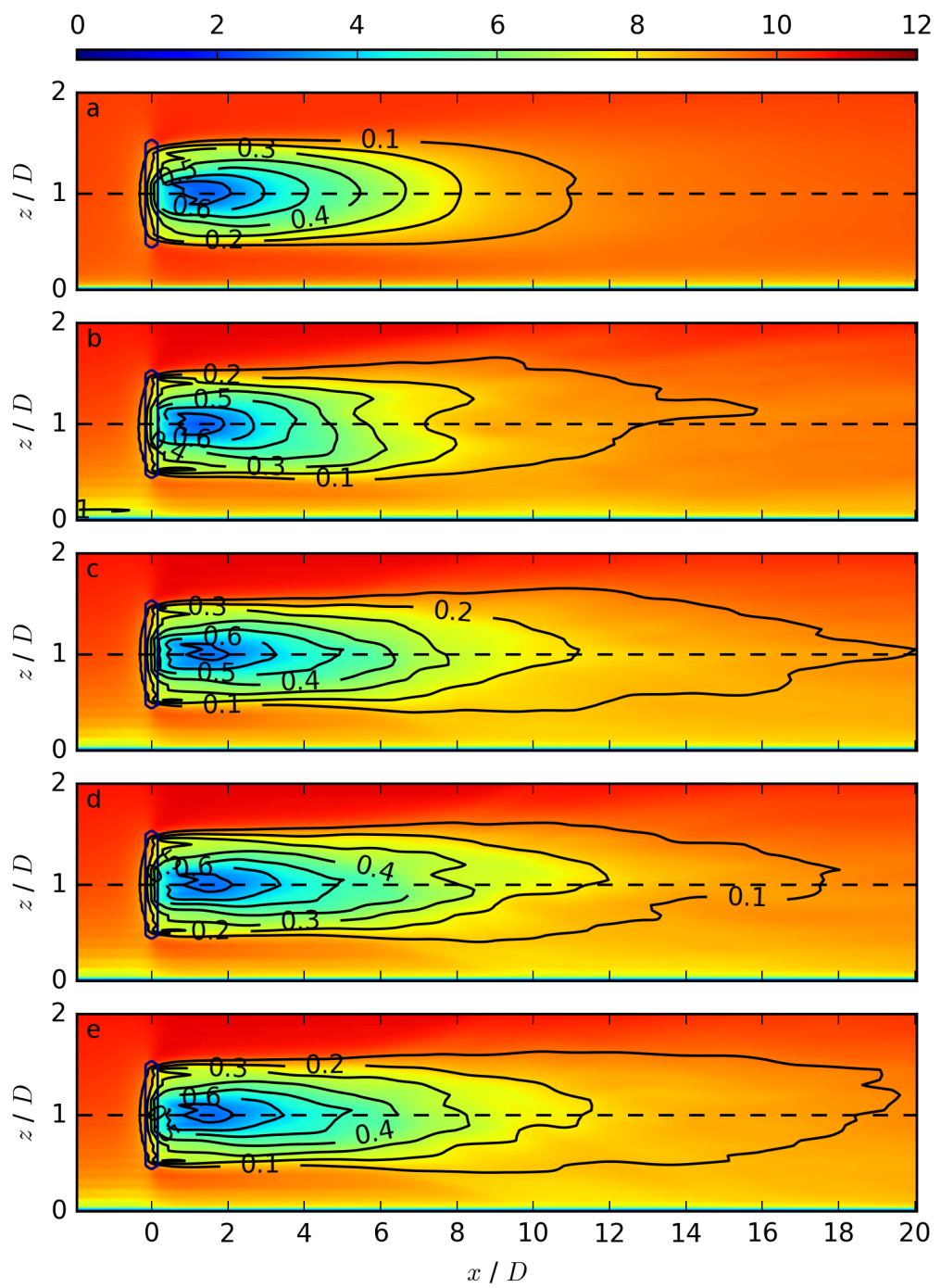

C

Fig. 6 Transitional vertical cross-sections of $\overline{u_{i, j_{0}, k}}$ in $\mathrm{m} \mathrm{s}^{-1}$ for the benchmark windturbine simulation in $(a)$, the reference wind-turbine simulation in $(b)$ and parametrization wind-turbine simulations of type $A, B$, and $C$ in $(c),(d)$, and $(e)$ respectively. The black contours represent $V D_{i, j_{0}, k}$. 



A

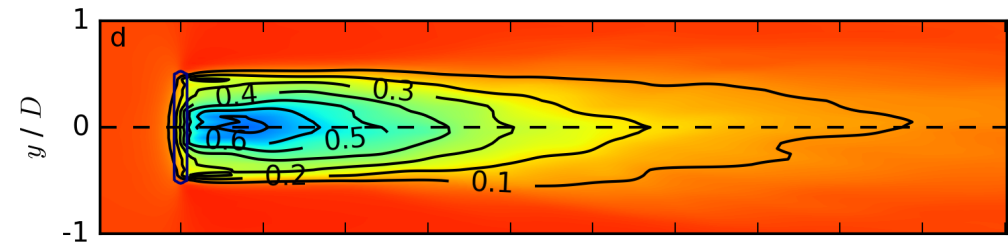

B

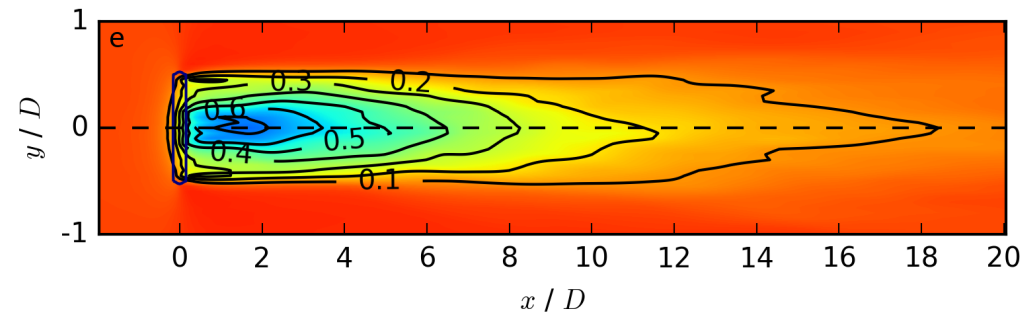

C

Fig. 7 Transitional horizontal cross-sections of $\overline{u_{i, j, k_{h}}}$ in $\mathrm{m} \mathrm{s}^{-1}$ for the benchmark windturbine simulation in $(a)$, the reference wind-turbine simulation in $(b)$ and parametrization wind-turbine simulations of type $A, B$, and $C$ in $(c),(d)$, and (e) respectively. The black contours represent $V D_{i, j, k_{h}}$. 

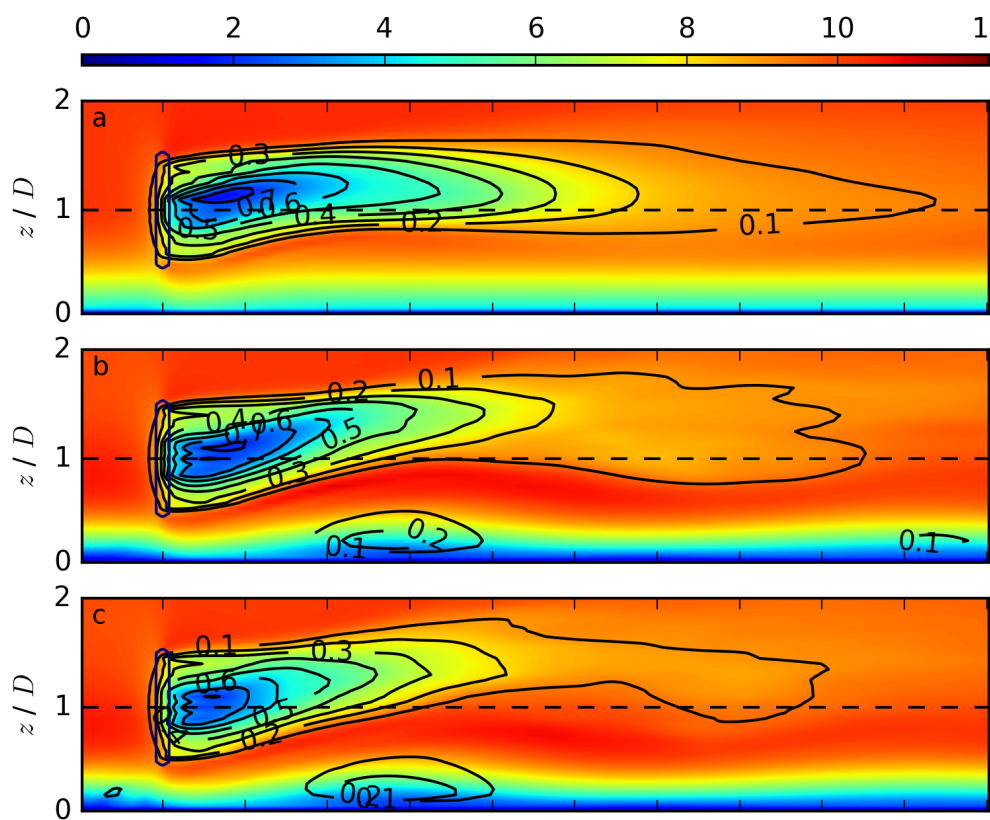

A

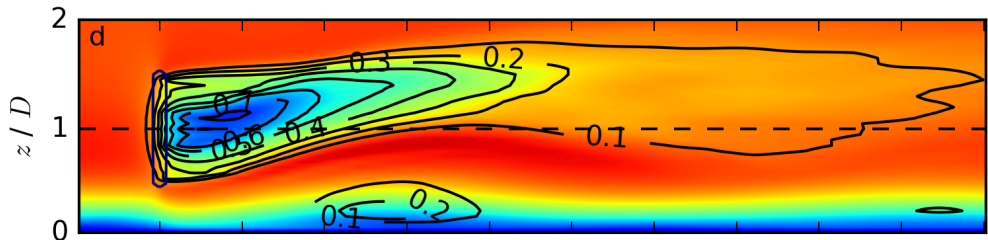

B

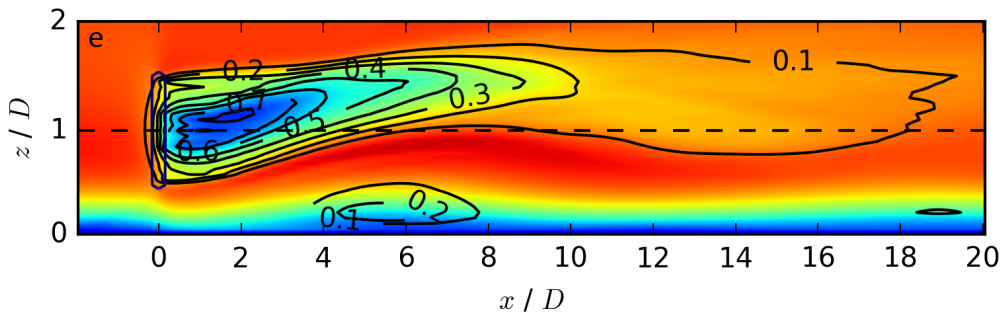

C

Fig. 8 Night-time vertical cross-sections of $\overline{u_{i, j_{0}, k}}$ in $\mathrm{m} \mathrm{s}^{-1}$ for the benchmark wind-turbine simulation in $(a)$, the reference wind-turbine simulation in $(b)$ and parametrization windturbine simulations of type $A, B$, and $C$ in $(c),(d)$, and $(e)$ respectively. The black contours represent $V D_{i, j_{0}, k}$. 

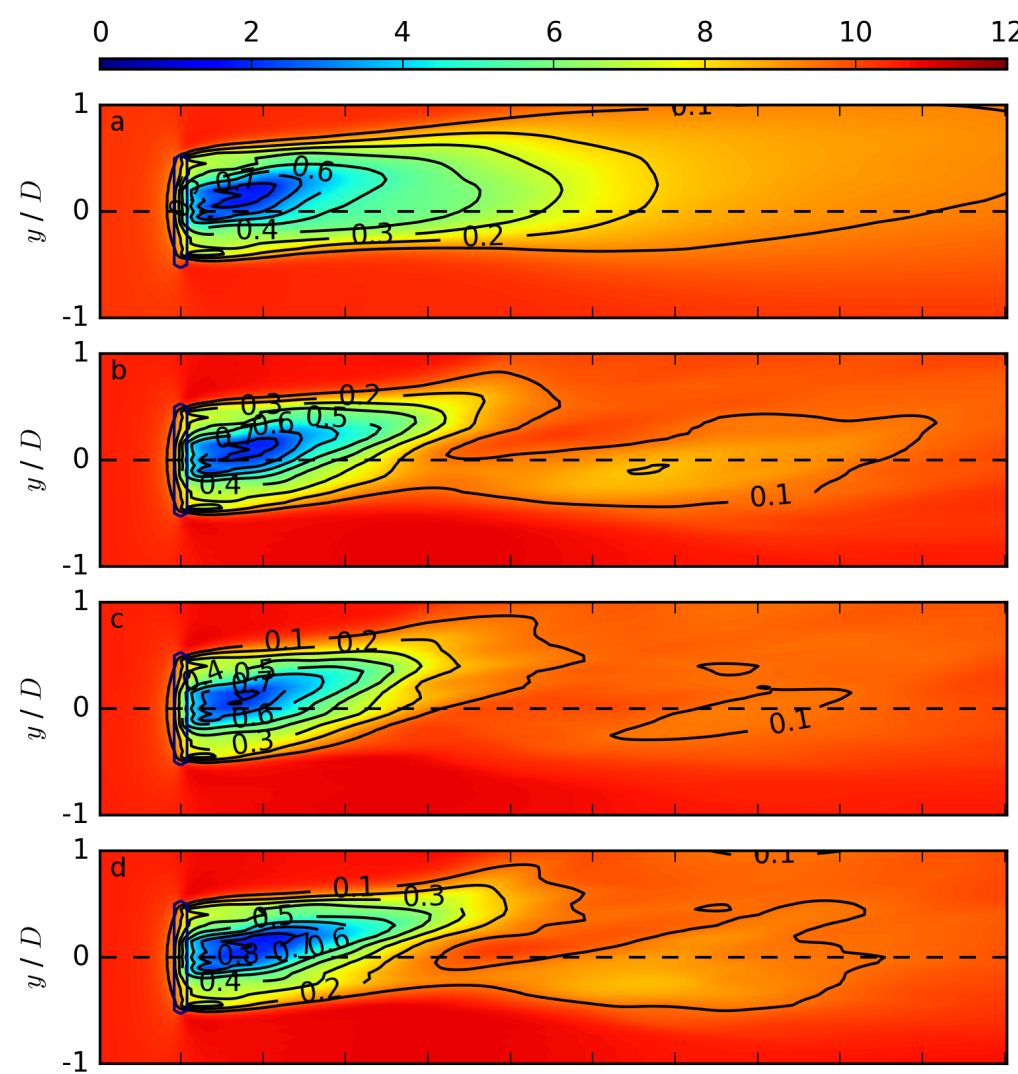

B

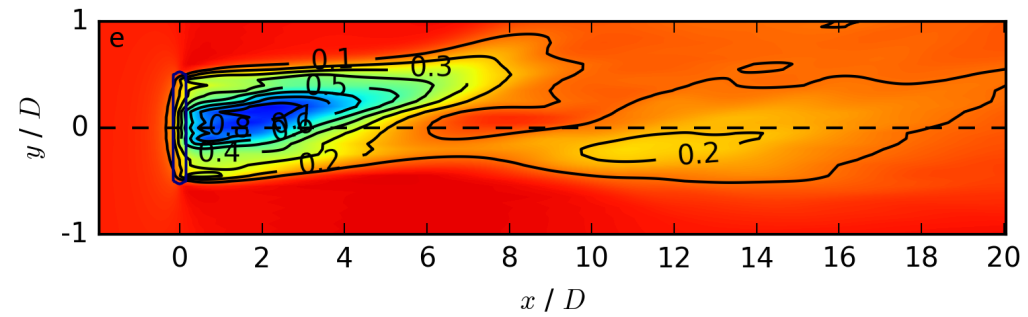

C

Fig. 9 Night-time horizontal cross-sections of $\overline{u_{i, j, k_{h}}}$ in $\mathrm{m} \mathrm{s}^{-1}$ for the benchmark windturbine simulation in $(a)$, the reference wind-turbine simulation in $(b)$ and parametrization wind-turbine simulations of type $A, B$, and $C$ in $(c),(d)$, and $(e)$ respectively. The black contours represent $V D_{i, j, k_{h}}$. 
the parametrization results in an investigation of the impact of the turbulence preserving method on the wake characteristics by applying perturbation velocities from the diurnal-cycle precursor simulation instead of applying the synchronized timestep data as inflow condition. In addition, the impact of the approximated background wind profiles is contrasted with the horizontal averaged profiles.

The wake structures between the reference and the benchmark wind-turbine simulations in Figs. 4-9a and b are quantitatively consistent. Especially for the transitional state, the agreement between the benchmark and the reference wind-turbine simulations is very good, whereas the wake recovers less rapidly during the day and more rapidly during the night. Further, there are differences in the wake-deflection angle. These differences can be attributed to the simplifications. Both aspects are described in detail in the following:

During the day, the less rapid recovery in the reference wind-turbine simulation results from the value of $\alpha_{0}$. A value of 0.5 instead of 0.7 for $\alpha_{0}$ decreases the background turbulence. The absence of the lateral wake deflection (Fig. 5b) can be attributed to the modification of using the same perturbation velocities for three consecutive timesteps. This was tested in an additional simulation that do not apply the same perturbation velocity for three consecutive timesteps. In this simulation, the streamwise wake extension was not completely conform with the benchmark wind-turbine simulation, however, the wake deflection was prevalent.

In the transitional period, the wake recovery at a certain downstream position is rather similar in the benchmark and in the reference wind-turbine simulations. The wake deflection from the benchmark wind-turbine simulation is also represented in the reference wind-turbine simulation. The smaller wake-deflection angle can be attributed to the stratification-dependent turbulent inflow method from Eq. 10 that uses the background turbulence of the evening ABL after $t=18 \mathrm{~h}$ in combination with $\alpha_{0}=0.5$ instead of synchronized diurnal-cycle data of the evening ABL from $t=18 \mathrm{~h}$ to $19 \mathrm{~h}$, which exhibits a stronger northwards wind component approaching $t=19 \mathrm{~h}$. Furthermore, the representation of the wake deflection in the reference wind-turbine simulation also reinforces our above reasoning of the different wake-deflection angle during the day.

During the night, the more rapid recovery in the reference wind-turbine simulation results from the relatively large value of $\alpha_{0}$ (0.5 instead of 0.3$)$, which increases the background turbulence. Other differences during the night represent the profile of the upstream velocity component, especially close to the ground, the flow pattern below the wake in between $4 D$ and $8 D$, and the upward bending of the wake with larger wind speed values between $6 D$ and $8 D$ (Fig. 8a and b). These differences can be related to a larger vertical gradient of the approximated $u_{e}$ and $v_{e}$ profiles with height (as shown in Fig. 1) in comparison to the stable ABL profile, which serves as background wind profile in the benchmark wind-turbine simulation. The approximated profiles further result in a larger veering of the wind, which causes a stronger wake deflection at hub height in the reference wind-turbine simulation (Fig. 9b). 
Table 3 List of the velocity-deficit deviation between two wind-turbine simulations for the day, the transition, and the night, as vertical and lateral average at a downstream position of $4 D$. The nomenclature $b$ corresponds to benchmark, $r$ to reference, $A$, $B$, and $C$ to parametrization of type $A, B$, and $C$.

\begin{tabular}{c|c|c|c|c|c|c} 
difference / & \multicolumn{2}{|c|}{ day } & \multicolumn{2}{c|}{ transition } & \multicolumn{2}{c}{ night } \\
\hline slice & vertical & lateral & vertical & lateral & vertical & lateral \\
\hline$b-r$ & 4.8 & 7.0 & 2.6 & 3.1 & 5.5 & 3.5 \\
\hline$r-A$ & 3.9 & 2.7 & 2.3 & 4.7 & 2.2 & 1.6 \\
\hline$A-B$ & 1.2 & 0.8 & 0.4 & 0.4 & 2.3 & 1.9 \\
\hline$B-C$ & 0.4 & 0.5 & 0.3 & 0.3 & 0.4 & 0.2 \\
\hline
\end{tabular}

The velocity-deficit deviations of the reference wind-turbine simulation from the benchmark wind-turbine simulations are listed in Table 3 as $(b-r)$ case for the day, the transition, and the night as vertical and lateral averages at a downstream position of $4 D$, corresponding roughly to the transition between the near and the far wake region. The values are calculated as the difference of the velocity deficit from Eq. 5 between the benchmark $(b)$ and the reference $(r)$ wind-turbine simulations at $4 D$. At day and night, the values are roughly twice as large as in the transitional state. This corresponds to the less (more) rapid wake recovery during the day (night), which results from the value of $\alpha_{0}$, modifying the background turbulent intensity. The differences in the wake-deflection angle are represented by the lateral values. The largest value reflects the much larger difference in the wake-deflection angle during the day in comparison to the transitional and nocturnal situation.

The presented comparison of the wake structures between the benchmark and the corresponding reference wind-turbine simulations reveal qualitatively and quantitatively consistent results for all three ABL regimes. The deviations can all be attributed to the applied simplifications in the reference windturbine simulation set-up. Consequently, the reference wind-turbine simulations are adequate representations of the benchmark wind-turbine simulations at day, in the transitional period, and at night. This enables us in the following to compare the parametrization wind-turbine simulations directly to the reference wind-turbine simulations, which is an important step towards the verification of the proposed parametrization.

6.2 Reference wind-turbine simulations and parametrization wind-turbine simulations of type $A$

As second step, the parametrization wind-turbine simulations of type $A$ are compared to the reference wind-turbine simulations to investigate the coupled impact of stratification-dependent weightings in Eq. 11 (Eqs. 12-14) and the perturbation velocities, which now result from a neutral ABL precursor simulation. The wake structures between the reference and the parametrization wind-turbine simulations of type $A$ in Figs. 4-9b and c are rather similar, with only minor differences: 
During the day and in the transitional period, the wake recovery is slightly less rapid for the parametrization wind-turbine simulations of type $A$, whereas at night, the wake recovers slightly more rapid.

The less rapid wake recovery during the day and the more rapid wake recovery during the night can be related to the background turbulence, imposed by the perturbation velocities resulting from the neutral ABL precursor simulation via Eq. 10, which is stronger (weaker) than the background turbulence of the stable ABL (convective ABL) in the night-time (daytime) reference windturbine simulation (Fig. 2a-c), resulting in a more (less) rapid wake recovery during night (day).

The contribution of the stable ABL and the convective ABL structure via the stratification-dependent weightings in Eq. 11 also accounts for the 3D turbulence structure. However, the impact on the wake structure is less pronounced than using the background turbulence of the corresponding diurnalcycle regime (stable ABL, convective ABL) in the reference wind-turbine simulations.

The wake structure difference is less pronounced in the evening ABL in comparison to the convective ABL. The magnitude of the wind perturbations (Fig. 2) are rather similar for the evening ABL and the neutral ABL, corresponding to a similar background turbulence intensity imposed in the evening ABL parametrization wind-turbine simulation in comparison to the convective ABL parametrization wind-turbine simulations.

Furthermore, the wake in the transitional period in Fig. 7c is no longer deflected. This effect is caused by the utilization of the evening ABL wind fields in combination with the neutral ABL wind fields, both contributing to the sustainment of background turbulence via the stratification-dependent weighting $\boldsymbol{\alpha}_{i^{*}, j, k}$, instead of applying only the evening ABL data, as it is the case in the reference wind-turbine simulation. This consideration of the evening ABL characteristics via the stratification-dependent weighting $\boldsymbol{\alpha}_{i^{*}, j, k}$ does not reproduce the wake deflection to the same extent. The simulated lateral wake deflection of the night-time reference wind-turbine simulation (Fig. 9b), however, is presented in the parametrization wind-turbine simulation (Fig. 9c), as this deflection is related to the applied background wind profiles $u_{e}$ and $v_{e}$ from Eqs. 8 and 9.

The velocity-deficit deviations of the parametrization wind-turbine simulations of type $A$ from the reference wind-turbine simulations are listed in Table 3 as $(r-A)$ for all regimes. In comparison to the $(b-r)$ differences, the $(r-A)$ values are in most cases much smaller (exception: lateral transitional slice). This is in agreement with the rather similar wake structures in comparison to the obvious wake structure differences between the benchmark and the reference wind-turbine simulations. The relatively large lateral velocity-deficit-deviation value in the transitional regime can be attributed to the wake deflection difference between the reference wind-turbine simulation and the type $A$ parametrization wind-turbine simulation as documented in Fig. $7 \mathrm{~b}$ and c. This effect is much less pronounced in the daytime (Fig. 5b and c), and, especially, in the nocturnal regimes (Fig. 9b and c), reflected in 
their corresponding lateral velocity-deficit-deviation values. Furthermore, the vertical value is larger during the day in comparison to the transitional period, resulting from the smaller neutral ABL background turbulence in comparison to the convective ABL background turbulence, whereas it is comparable to the evening ABL background turbulence. The vertical value at night is similar to the transitional one, even the wake recovers slightly more rapidly at night in comparison to the less rapid recovery in the transitional period. This same sign results from the use of absolute velocity-deficit values in the calculation of the averaged differences. However, as the tendency towards a more or less rapid recovery is persistent in the whole wake, the absolute values are verified.

The comparison of the wake structures between the reference and the corresponding parametrization wind-turbine simulations with function set of type $A$ also reveals qualitatively and quantitatively consistent results for all three ABL regimes. The deviations can all be attributed to the usage of the flow field from the neutral ABL precursor simulation to sustain the background turbulence in combination with the stratification-dependent parameters.

\subsection{Parametrization wind-turbine simulations of type $A, B$, and $C$}

As third step, the parametrization wind-turbine simulations of type $B$ (vertical profiles from Eq. 15) are compared to the type $A$ parametrization wind-turbine simulations, which can be considered as the most precise parametrization windturbine simulations. Furthermore, the parametrization wind-turbine simulations of type $C$ are compared to the type $B$ parametrization wind-turbine simulations, to investigate the impact of the additional simplification of the stratification-dependent weighting $\boldsymbol{\alpha}_{i^{*}, j, k}$ to three scalar quantities. The wake structures between the parametrization wind-turbine simulations of type $A$ and $B$ are rather similar, only the extent of the wake recovery downstream of the wind turbine differs. In particular, the wake recovers more rapidly in the daytime regime and less rapidly in the night-time regime for parametrization type $B$. In the transitional regime, the difference is marginal. The less rapid recovery during the night can be related to the removal of horizontal variability provided by the three 3D matrices $\boldsymbol{\alpha}_{i^{*}, j, k}$ (Eq. 11) of type $A$ in comparison to the three vertical $1 \mathrm{D}$ vectors of type $B$. The more rapid recovery during the day is contradictory to this explanation. However, it can be explained with the larger magnitude of $\mathbf{u}_{\alpha_{k}}$ (three $1 \mathrm{D}$ vectors used in parametrization $\mathrm{B}$ ) in comparison to $\mathbf{u}_{\alpha_{i}}$ and $\mathbf{u}_{\alpha_{j}}$ (not shown here).

The deviation of the type $B$ parametrization wind-turbine simulations from type $A$ are listed in Table 3 as $(A-B)$. The tendencies of the more or less rapid wake recovery during the day and the night result in similar sized vertical and lateral velocity-deficit-deviation values. Both transitional values are much smaller than the daytime or night-time values and are influenced by the marginal wake structure difference as shown in Figs. 6 and 7.

The wake structure differences between the parametrization wind-turbine simulations of type $B$ and $C$ are only marginal in the $x-y$ cross-section at hub 
height, as the values $\alpha_{u}, \alpha_{v}$, and $\alpha_{w}$ correspond to hub height values of $\mathbf{u}_{\alpha_{k}}$. In the $x-z$ cross-sections through $y_{0}$, the wake recovers slightly less rapidly at night. This can, again, be related to the removal of vertical gradients in the stratification-dependent weighting $\boldsymbol{\alpha}_{i^{*}, j, k}$. In the transitional period and during the day, the differences are marginal.

Furthermore, the deviations of type $C$ parametrization wind-turbine simulations from type $B$ are listed in Table 3 as $(B-C)$. The lateral and vertical velocity-deficit-deviation values are rather small for all regimes. This corresponds to the marginal differences between parametrization type $B$ and $C$ in Figs. 4-9. Small lateral values at hub height are expected, as the values $\alpha_{u}$, $\alpha_{v}$, and $\alpha_{w}$ from the parametrization of type $C$ are also hub height values. The small vertical values, however, indicate the feasibility of parametrization of type $C$ to represent the stratification-dependent turbulent flow through a wind turbine.

The comparison of the wake structures between the parametrization windturbine simulations of type $A$ and type $B$ and likewise of type $B$ and type $C$ reveal qualitatively and quantitatively consistent results for all three ABL regimes. The deviations can all be attributed to the simplifications applied to the stratification-dependent weightings $\boldsymbol{\alpha}_{i^{*}, j, k}$.

\subsection{Streamwise velocity ratio and total turbulent intensity}

The parametrization effect on the streamwise velocity-ratio profiles $V R$ from Eq. 5 and the total turbulence intensity profiles $I$ from Eq. 6 are shown in Fig. 10 for the three ABL regimes. During the day, the $V R$ profiles (in $a$ ) are almost overlapping for the reference wind-turbine simulation and for the parametrization wind-turbine simulations of type $A, B$, and $C$. In the transitional state (in $c$ ), a slightly less rapid wake recovery prevails, compared to the reference simulations. Care must be taken when interpreting the streamwise $V R$ through the centre of the rotor at night (in $e$ ) because of the lateral wake deflection.

Considering the profiles of $I$, the parametrization wind-turbine simulations of type $A, B$, and $C$ are also in good agreement with the reference wind-turbine simulations for the day and the transitional state (in $b$ and $d$ ). At night (in $f$ ), the values of $I$ are slightly larger for the parametrization wind-turbine simulation of type $A$ in comparison to type $B$ and $C$, which correlates with the slightly more rapid wake recovery depicted in Figs. 8 and 9.

Therefore, the modified turbulence preserving method results in a significant improvement of the profiles of $V R$ and $I$, especially regarding the turbulent intensity. This conclusion is valid for the reference case as well as for all function sets applied for the stratification-dependent weightings $\boldsymbol{\alpha}_{i^{*}, j, k}$. 

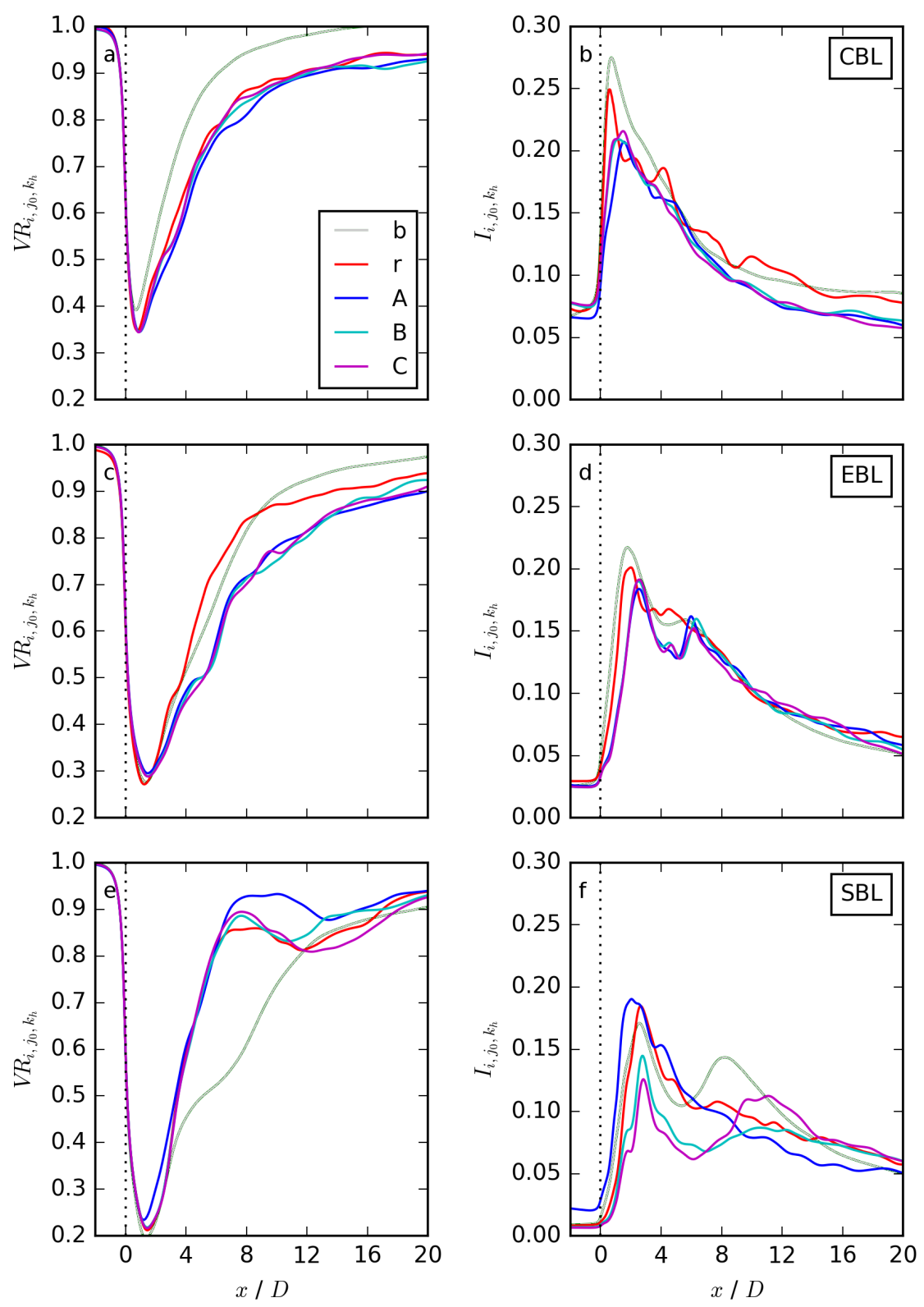

Fig. 10 Streamwise dependency of $V R_{i, j_{0}, k_{h}}$ in $(a),(c)$, and $(e)$ as well as $I_{i, j_{0}, k_{h}}$ in $(b)$, $(d)$ and $(f)$ for all wind-turbine simulations representing the daytime situation in $(a)$ and $(b)$, the transition in $(c)$ and $(d)$ and the night-time situation in $(e)$ and $(f)$. The subscript 'b' corresponds to benchmark and 'r' to reference. 
6.5 Summary

The investigation of the numerical simulation results using the developed parametrization reveals a good qualitative and quantitative agreement of type $A$, $B$, and $C$ simulations with their corresponding reference wind-turbine simulations and also with the benchmark wind-turbine simulations. The only major limitation of the parametrization wind-turbine simulations is the too weak representation of the wake deflection in comparison to the reference wind-turbine simulations. This deviation is primarily related to the use of 3D wind fields of $u, v$, and $w$ of the neutral ABL precursor simulation in stationary equilibrium.

Type $C$ is the most simple and most effective parametrization that shows very small velocity-deficit deviations. The velocity-deficit-deviation values from Table 3 are calculated at a downstream position of $4 D$. A velocity-deficitdeviation calculation considering the complete downstream region decreases the absolute deviation values. However, the same relative velocity-deficit deviation persists. Therefore, the vertical and lateral values at $4 D$ are suitable as representations of the whole wake, reinforcing the preference of the parametrization with function set $C$.

This simple parametrization with function set of type $C$ results in numerically very efficient wind-turbine simulations for different thermal stratifications. The only requirements are 3D wind fields of $u, v$, and $w$ of a neutral ABL precursor simulation, stratification-dependent values $\alpha_{u}, \alpha_{v}$, and $\alpha_{w}$, and appropriate background wind profiles $u_{e}, v_{e}$, and $w_{e}$.

Our proposed approach to simulate stratification-dependent wind-turbine flow by LES reduces the computational costs from 14 days, required for one diurnal-cycle simulation of the idealized ABL and the corresponding four benchmark wind-turbine simulations, to less than one hour, each time performed on 256 Intel Xeon E5-2697 v3 threads at $2.6 \mathrm{GHz}$.

Summarizing these results, it can be state that due to the simplicity of providing stratification-dependent turbulent inflow fields from Englberger and Dörnbrack (2017b) in a modified version of the turbulence preserving method (Englberger and Dörnbrack, 2017a), our parametrization results in a computationally fast, simple, and efficient tool for analyzing the wake characteristics of a wind turbine in a turbulent ABL flow under different thermal stratifications. Further, it can be used as simplification for many different applications e.g. individual wake characteristics, optimized wind-farm set-ups, providing an alternative and fast testbed for stratification-dependent LES of wind-turbine wakes compared to complete diurnal-cycle simulations and the corresponding synchronized wind-turbine simulations, which requires turbulence data as input at each timestep.

\section{Conclusion}

The wake characteristics of a wind turbine in a turbulent ABL flow were investigated by means of large-eddy simulation for different thermal stratifications. 
Therefore, a modified version of the turbulence preserving method from Englberger and Dörnbrack (2017a) was developed and applied.

The consideration of three wind fields of $u, v$, and $w$ of a neutral ABL precursor simulation, a stratification-dependent weighting, and appropriate background wind profiles made this approach applicable for various atmospheric conditions occurring throughout the course of a day. Only the three wind fields of an equilibrium neutral ABL state sustain the turbulent inflow in wind-turbine simulations with open horizontal boundary conditions, making it a simple and computationally fast approach. The additional consideration of the stratification-dependent weighting in combination with appropriate background wind profiles, further, makes it a numerically effective approach, which is able to accounts for different atmospheric conditions. The simplest function set (type $\mathrm{C}$ ) of the stratification-dependent parameters consists of only three values $\alpha_{u}, \alpha_{v}$, and $\alpha_{w}$. Surprisingly, these three scalars are sufficient to reproduce the atmospheric daytime, transition, and night-time situation of the synchronized diurnal-cycle benchmark wind-turbine simulations. With the simplest approach, no diurnal-cycle precursor simulation is needed, the values $\alpha_{u}, \alpha_{v}$, and $\alpha_{w}$ can be approximated for different atmospheric situations by taking the values as suggested in this work. This reduces the computational costs by a factor of $\mathcal{O}\left(10^{2}\right)$, still providing a sufficient approximation of the expected wake structure. The parametrization further offers a suitable testbed for extensive sensitivity studies using a large range of parameters, like different rotor configurations, different subgrid-scale models, different atmospheric conditions, ranging from very stable, to stable, to near-neutral, to convective, to very convective (by varying $\alpha_{u}, \alpha_{v}$, and $\alpha_{w}$ ), or different background wind profiles (by varying the fit used for $u_{e}, v_{e}$, and $w_{e}$ ) and can be applied to develop appropriate wind-farm set-ups in the future. All applications are aiming at maximizing the power production and minimizing the fatigue loading.

The representation of all atmospheric states is crucial for studying the interaction of the ABL flow with a wind turbine. Especially, if we take into account that the near-neutral stratification of the transitional period, which was used in most of previous numerical wind-turbine studies, occurs for example only with a frequency of roughly $10 \%$ according to data from the field experiment SWiFT (Facility Representation and Preparedness; 730 days of measurement in the period from 2012 to 2014) (Kelley and Ennis, 2016). Our proposed parametrization of the stratification-dependent turbulent inflow, developed and tested in this paper, offers a simple, numerically efficient, and computationally fast large-eddy simulation approach, which meets these requirements.

Acknowledgements The authors thank Fernando Porté-Agel and Gert-Jan Steeneveld for the constructive discussion on the turbulence preserving method. Further, we thank Mark Zagar for providing the airfoil data of the 10-MW reference wind turbine from DTU. TThis work was performed within the project LIPS, funded by the Federal Ministry of Economy and Energy on the basis of a resolution of the German Bundestag under the contract number 0325518. The authors gratefully acknowledge the Gauss Centre for Supercomputing e.V. 
(www.gauss-centre.eu) for funding this project by providing computing time on the GCS Supercomputer SuperMUC at Leibniz Supercomputing Centre (LRZ, www.lrz.de).

\section{Appendix 1: Precursor simulations}

Neutral atmospheric boundary-layer precursor simulation

In the neutral ABL precursor simulation (Englberger and Dörnbrack, 2017a), performed with periodic horizontal boundary conditions, an additional forcing $-u_{*}^{2} / H$ was applied for the zonal wind component $u$ of Eq. 1 to drive the neutral ABL flow, by using a friction velocity $u_{*}=0.4 \mathrm{~m} \mathrm{~s}^{-1}$ and $H$ corresponding to the height of the computational domain. The initial wind speed was set to zero, and the drag coefficient in the surface parametrization was set to 0.1. A more detailed description of this neutral ABL precursor simulation can be found in Englberger and Dörnbrack (2017a).

\section{Diurnal-cycle precursor simulation}

The idealized diurnal-cycle simulation (Englberger and Dörnbrack, 2017b) offers the convective $\mathrm{ABL}$, the evening $\mathrm{ABL}$, the stable $\mathrm{ABL}$, and the morning $\mathrm{ABL}$ as representatives for different atmospheric situations that occur in the course of a day. It was performed over homogeneous surface with periodic horizontal boundary conditions on $512 \times 512$ grid points in the horizontal with a resolution of $5 \mathrm{~m}$. The domain height was $2 \mathrm{~km}$ with a vertical resolution of $5 \mathrm{~m}$ in the lowest $200 \mathrm{~m}, 10 \mathrm{~m}$ up to $800 \mathrm{~m}$, and $20 \mathrm{~m}$ approaching the domain top. The simulation was initialised with a geostrophic wind of $u=u_{e}=10 \mathrm{~m} \mathrm{~s}^{-1}$ in zonal (east - west, streamwise) direction and zero for the meridional (north south, spanwise, lateral) $\left(v=v_{e}=0\right)$ and vertical $\left(w=w_{e}=0\right)$ wind components. The initial potential temperature of $300 \mathrm{~K}$ was constant up to $1 \mathrm{~km}$ and increased linearly with height above according to a lapse rate of $10 \mathrm{~K} \mathrm{~km}^{-1}$. The prescribed temporal evolution of the sensible heat flux used in the dry idealized ABL simulation at the surface corresponds to the square of a sinus profile at day with a maximum of $140 \mathrm{~W} \mathrm{~m}^{-2}$ at noon and a constant flux of $-10 \mathrm{~W} \mathrm{~m}^{-2}$ during the night. A more detailed description of this idealized diurnal-cycle simulation can be found in Englberger and Dörnbrack (2017b).

\section{Wind-field structure}

Snapshots of the instantaneous wind fields $u, v$, and $w$ of the neutral ABL precursor simulation, as well as of the convective ABL, the evening ABL, the stable ABL, and the morning ABL regimes of the idealized diurnal-cycle simulation, are presented in Fig. 11, with the following characteristics: 
The neutral ABL exhibits a shallow ABL with low vertical wind speeds. 880 In the convective ABL, the ABL reaches higher altitudes with significant hor${ }_{881}$ izontal and vertical winds. Further, the boundary-layer flow consists of larger ${ }_{882}$ turbulent eddies in comparison to the other stratifications, which results from the maximum of positive buoyancy, induced by the surface heat flux. In the evening ABL, the convective updrafts as well as the horizontal wind weakens. In the stable ABL, a low-level jet starts to develop with a wind turning with and no significant vertical wind. The situation in the morning ABL is very similar to the stable ABL with an intensification of the low-level jet.

\section{Derivation of background wind profiles}

From the five characteristic states of the neutral ABL, the convective ABL, the evening ABL, the stable ABL, and the morning ABL, respectively, horizontal means of $u$ and $v$ can be calculated (Fig. 1a and $\mathrm{b}$ ). The zonal and meridional background wind fields of the convective ABL and the evening ABL are fundamentally different in comparison to the stable ABL and the morning ABL. In the convective $\mathrm{ABL}$ and in the evening $\mathrm{ABL}$, the vertical wind shear of the zonal wind is rather small above the ground and the meridional wind is nearly zero. In the stable ABL and in the morning ABL, the vertical wind shear of the zonal and meridional wind is very pronounced, with a supergeostrophic wind maximum prevailing in the morning ABL. The different profiles of the stable ABL and the morning ABL (in contrast to the convective ABL and the evening $\mathrm{ABL}$ ) are influenced by the Coriolis force, which dominates the buoyancy effects at night and results in the Ekman spiral. A more detailed description of the convective ABL, the evening ABL, the stable ABL, and the morning ABL wind conditions are given in Englberger and Dörnbrack (2017b). Fits on these vertical profiles (Fig. 1a and b) are used as background wind profiles $\left(u_{e}, v_{e}\right)$ for the wind-turbine simulations, performed with the proposed parametrization. The applied fits are listed in Sect. 3 for a daytime (convective $\mathrm{ABL}$, evening $\mathrm{ABL}$ ) and a night-time (stable ABL, morning ABL) situation.

\section{Appendix 2: Turbulence Preserving Method}

\section{Original version}

The original version of the turbulence preserving method imposes extracted velocity perturbations from a neutral ABL precursor simulation as an additional force in the whole computational domain. The perturbation velocities $\left.\mathbf{u}_{p}^{*}\right|_{i, j, k} ^{\xi}$ were extracted from the neutral ABL precursor simulation according 
to

$$
\left.\mathbf{u}_{p}^{*}\right|_{i, j, k} ^{\xi}=\alpha \beta \underbrace{(\left.\mathbf{u}_{p}\right|_{i^{*}, j, k}-\underbrace{\left.\frac{1}{n m} \sum_{i=1}^{n} \sum_{j=1}^{m} \mathbf{u}_{p}\right|_{i, j, k}}_{I I})}_{I},
$$

where $\left.\mathbf{u}_{p}\right|_{i^{*}, j, k}$ is the velocity vector of a neutral ABL equilibrium state and the term $I I$ in Eq. 16 denotes the horizontal average of the mean value of the corresponding wind component at each grid point $i, j$, and $k$. The indices of the grid points are denoted by $i=1 \ldots n, j=1 \ldots m$, and $k=1 \ldots l$ in the $x, y$, and $z$ directions, respectively.

The perturbation velocities from Eq. 16 modify the velocity field of the wind-turbine simulation $\left.\mathbf{u}\right|_{i, j, k} ^{\xi}$ at the initial timestep $\xi=0$ and at each following timestep $\xi$. The values $\left.\mathbf{u}_{p}\right|_{i^{*}, j, k}$ of the precursor simulation were shifted in the streamwise direction by one grid point every timestep $\xi$, symbolized by $i^{*}=i+\xi^{*}$, with $i^{*} \in[1, n]$ and $\xi^{*}$ representing the number of timesteps since the start of the simulation. Furthermore, the difference as denoted by $I$ in Eq. 16 was multiplied with a random number $\beta$ ranging from -0.5 to 0.5 . Both, the grid point shift and the random number multiplication, were necessary to only apply the spectral energy distribution of the precursor simulation instead of impressing individual flow patterns onto the wind-turbine simulation. To account for different magnitudes of the background turbulence, the term $I$ in Eq. 16 was additionally multiplied by a factor $\alpha$, representing the amplitude of the turbulence perturbations. Numerical simulations of the turbulent flow through a wind turbine performed with this original version of the turbulence preserving method in Englberger and Dörnbrack (2017a) resulted in realistic wake structures, which were quantitatively comparable with observations and other numerical simulation results.

\section{Modified version}

A comparison of the streamwise velocity ratio $V R_{i, j_{0}, k_{h}}$ and the turbulent intensity $I_{i, j_{0}, k_{h}}$ between wind-turbine simulations performed with the original version of the turbulence preserving method for $\alpha=1$ (B_1), $\alpha=5$ (B_5), and $\alpha=10$ (B_10) from Englberger and Dörnbrack (2017a) and synchronized diurnal-cycle wind-turbine simulations for the convective ABL, the evening $\mathrm{ABL}$, the stable $\mathrm{ABL}$, and the morning $\mathrm{ABL}$ regime from Englberger and Dörnbrack (2017b) is shown in Fig. 12.

The velocity ratio is comparable to the values of the stable ABL and the morning ABL wind-turbine simulations for $\alpha=1$ (simulation B_1). For $\alpha=5$, the values of simulation B_5 are comparable to the evening ABL windturbine simulation and for $\alpha=10$ (simulation B_10) to the convective ABL wind-turbine simulation. The $V R_{i, j_{0}, k_{h}}$-gradient of the wake recovery is only 
slightly smaller in the near wake $(x \leq 5 D)$. The turbulent intensities for all synchronized wind-turbine simulations compare quantitatively very well with the B_1 simulation with $\alpha=1$. However, for $\alpha=5$ and $\alpha=10$, the turbulent intensities of simulations B_5 and B_10 are too large in comparison to all synchronized diurnal-cycle wind-turbine simulations. The pronounced deviations of the base case simulations (B_1, B_5, B_10) from the synchronized ones call for an optimization of the original version of the turbulence preserving method, which considers the dependence of the thermal stratification of the ABL in a new, modified version.

Various tests studying the sensitivity of the numerical results to the choice of $\alpha \cdot \beta$ revealed that the random number $\beta$, impressed at every timestep in the whole domain, destroys the energy spectra of the neutral ABL and therefore the turbulence structure included in $I$, resulting in a synthetic turbulence structure with different amplitudes of the turbulence in B_1, B_5, and B_10. This problem has been solved in the modified version of the turbulence preserving method by eliminating $\beta$, resulting in a more rapid wake recovery and smaller values of the turbulent intensity for increasing $\alpha$. This was shown in Sect. 6 .

In addition, the factor $\alpha$ from Eq. 16 has been adapted in the new, modified version of the turbulence preserving method: We apply an adjustable value $\alpha_{0}$, which is adjusted to compare the synchronized diurnal-cycle wind-turbine simulations from Englberger and Dörnbrack (2017b) and the wind-turbine simulations performed herein. In more general applications of this approach (e.g. no resimulation of a certain situation), $\alpha_{0}$ can be set to 1 . To account for the different atmospheric states during the diurnal cycle, we further apply stratification-dependent weighting parameters $\boldsymbol{\alpha}_{i, j, k}$, which represent the magnitude of the velocity perturbations. Their values are extracted from the corresponding situation of the diurnal-cycle simulation over homogeneous surface from Englberger and Dörnbrack (2017b). The modification of the turbulence preserving method from Eq. 16 can be summarized as

$$
\left.\mathbf{u}_{p}^{*}\right|_{i=1, j, k} ^{\xi}=\alpha_{0} \boldsymbol{\alpha}_{i^{*}, j, k} \underbrace{\left(\left.\mathbf{u}_{p}\right|_{i^{*}, j, k}-\left.\frac{1}{n m} \sum_{i=1}^{n} \sum_{j=1}^{m} \mathbf{u}_{p}\right|_{i, j, k}\right)}_{I} .
$$

The term inside the brackets of Eq. 17 corresponds to $I$ in Eq. 16. Furthermore, by changing the parametrization to a stability-dependent inflow condition, the perturbation velocities $\left.\mathbf{u}_{p}^{*}\right|_{i=1, j, k} ^{\xi}$ are only applied at the first grid point in $x$-direction. This modification was necessary, as a permanent impression of the perturbation velocities in the whole domain resulted in large positive and negative tendencies, which were compensated in the original version of the turbulence preserving method in Eq. 16 by the imposed randomness through $\beta$. 
References

Abkar M, Porté-Agel F (2014) The effect of atmospheric stability on wind-turbine wakes: A large-eddy simulation study. J Phys Conf Ser 524(1):012,138, DOI 10.1088/1742-6596/524/1/012138

Abkar M, Sharifi A, Porté-Agel F (2016) Wake flow in a wind farm during a diurnal cycle. J Turbul 17(4):420-441, DOI 10.1080/14685248.2015.1127379

Aitken ML, Kosović B, Mirocha JD, Lundquist JK (2014) Large eddy simulation of wind turbine wake dynamics in the stable boundary layer using the Weather Research and Forecasting model. J Renew Sust Energy 6:1529-1539

Bhaganagar K, Debnath M (2014) Implications of stably stratified atmospheric boundary layer turbulence on the near-wake structure of wind turbines. Energies 7(9):5740-5763, DOI 10.3390/en7095740

Bhaganagar K, Debnath M (2015) The effects of mean atmospheric forcings of the stable atmospheric boundary layer on wind turbine wake. J Renew Sust Energy 7(1):013,124, DOI 10.1063/1.4907687

Chamorro LP, Porté-Agel F (2009) A wind-tunnel investigation of windturbine wakes: Boundary-layer turbulence effects. Boundary-Layer Meteorol 132:129-149

Dörenkämper M, Witha B, Steinfeld G, Heinemann D, Kühn M (2015) The impact of stable atmospheric boundary layers on wind-turbine wakes within offshore wind farms. J Wind Eng Ind Aerodyn 144:146-153

Doyle JD, Gaberšek S, Jiang Q, Bernardet L, Brown JM, Dörnbrack A, Filaus E, Grubišic V, Kirshbaum DJ, Knoth O, Koch S, Schmidli J, Stiperski I, Vosper S, Zhong S (2011) An intercomparison of t-rex mountain-wave simulations and implications for mesoscale predictability. Mon Weather Rev 139:2811-2831

Englberger A, Dörnbrack A (2017a) Impact of neutral boundary-layer turbulence on wind-turbine wakes: A numerical modelling study. Boundary-Layer Meteorol 162(3):427-449

Englberger A, Dörnbrack A (2017b) Impact of the diurnal cycle of the atmospheric boundary layer on wind-turbine wakes: A numerical modelling study. Boundary-Layer Meteorol 166(3):423-448

Fröhlich J (2006) Large Eddy Simulation turbulenter Strömungen. Teubner Verlag / GWV Fachverlage GmbH, Wiesbaden, 414 pp (in German)

Grimsdell AW, Angevine WM (2002) Observations of the afternoon transition of the convective boundary layer. J Appl Meteorol 41(1):3-11, DOI 10.1175/1520-0450(2002)041;3C0003:OOTATO ¿3E2.0.CO;2

Kelley CL, Ennis BL (2016) Swift site atmospheric characterization. Tech. rep., Sandia National Laboratories (SNL-NM), Albuquerque, NM, USA.

Kühnlein C, Smolarkiewicz PK, Dörnbrack A (2012) Modelling atmospheric flows with adaptive moving meshes. J Comput Phys 231:2741-2763

Lee JC, Lundquist JK (2017) Observing and simulating wind-turbine wakes during the evening transition. Boundary-Layer Meteorol 164(3):449-474

Mann J (1994) The spatial structure of neutral atmospheric surface-layer turbulence. J Fluid Mech 273:141-168 
Margolin LG, Smolarkiewicz PK, Sorbjan Z (1999) Large-eddy simulations of convective boundary layers using nonoscillatory differencing. Phys D Nonlin Phenom 133(1):390-397

Medici D, Alfredsson PH (2006) Measurements on a wind turbine wake: 3D effects and bluff body vortex shedding. Wind Energy 9:219-236

Mirocha J, Kirkil G, Bou-Zeid E, Chow FK, Kosović B (2013) Transition and equilibration of neutral atmospheric boundary layer flow in one-way nested large-eddy simulations using the weather research and forecasting model. Mon Weather Rev 141:918-940

Mirocha J, Kosovic B, Aitken M, Lundquist J (2014) Implementation of a generalized actuator disk wind turbine model into the weather research and forecasting model for large-eddy simulation applications. J Renew Sust Energy 6:013104

Muñoz-Esparza D, Kosović B, Mirocha J, van Beeck J (2014) Bridging the transition from mesoscale to microscale turbulence in numerical weather prediction models. Boundary-Layer Meteorol 153:409-440

Naughton JW, Heinz S, Balas M, Kelly R, Gopalan H, Lindberg W, Gundling C, Rai R, Sitaraman J, Singh M (2011) Turbulence and the isolated wind turbine. In: 6th AIAA Theoretical Fluid Mechanics Conference, 27-30 June 2011, Honolulu, Hawaii, pp 1-19.

Prusa JM, Smolarkiewicz PK, Wyszogrodzki AA (2008) EULAG, a computational model for multiscale flows. Comput Fluids 37:1193-1207

Sathe A, Mann J, Barlas T, Bierbooms W, Bussel G (2013) Influence of atmospheric stability on wind turbine loads. Wind Energy 16(7):1013-1032, DOI $10.1002 /$ we. 1528

Schmidt H, Schumann U (1989) Coherent structure of the convective boundary layer derived from large-eddy simulations. J Fluid Mech 200:511-562, DOI $10.1017 /$ S0022112089000753

Shapiro A, Fedorovich E (2010) Analytical description of a nocturnal low-level jet. Q J R Meteorol Soc 136(650):1255-1262

Smolarkiewicz PK, Charbonneau P (2013) EULAG, a computational model for multiscale flows: An MHD extension. J Comput Phys 236:608-623

Smolarkiewicz PK, Dörnbrack A (2008) Conservative integrals of adiabatic durran's equations. Int J Numer Method Fluid 56:1513-1519

Smolarkiewicz PK, Margolin LG (1993) On forward-in-time differencing for fluids: extension to a curviliniear framework. Mon Weather Rev 121:18471859

Smolarkiewicz PK, Margolin LG (1998) MPDATA: a finite-difference solver for geophysical flows. J Comput Phys 140:459-480

Smolarkiewicz PK, Prusa JM (2002) Forward-in-time differencing for fluids: simulation of geophysical turbulence. In: Turbulent Flow Computation, Kluwer Academic Publishers, Boston, USA, pp 279-312

Smolarkiewicz PK, Prusa JM (2005) Towards mesh adaptivity for geophysical turbulence: continuous mapping approach. Int J Numer Method Fluid 47:789-801 
Smolarkiewicz PK, Pudykiewicz JA (1992) A class of semi-Lagrangian approximations for fluids. J Atmos Sci 49:2082-2096

Smolarkiewicz PK, Sharman R, Weil J, Perry SG, Heist D, Bowker G (2007) Building resolving large-eddy simulations and comparison with wind tunnel experiments. J Comput Phys 227:633-653

Stull RB (1988) An Introduction to Boundary Layer Meteorology. Kluwer Academic, Dordrecht, Netherlands

Troldborg N, Sørensen JN, Mikkelsen R (2007) Actuator line simulation of wake of wind turbine operating in turbulent inflow. J Phys Conf Ser 75:012,063

Vanderwende B, Lundquist JK (2012) The modification of wind turbine performance by statistically distinct atmospheric regimes. Environ Res Lett $7(3): 034,035$

Wedi NP, Smolarkiewicz PK (2004) Extending Gal-Chen and Somerville terrain-following coordinate transformation on time-dependent curvilinear boundaries. J Comput Phys 193:1-20

Wedi NP, Smolarkiewicz PK (2006) Direct numerical simulation of the plumbMcEwan laboratory analog of the QBO. J Atmos Sci 63:3226-3252

Wharton S, Lundquist JK (2012) Atmospheric stability affects wind turbine power collection. Environ Res Lett 7(1):014,005

Witha B, Steinfeld G, Heinemann D (2014) Wind energy - Impact of Turbulence, Springer, Oldenburg, Germany, chap High-resolution offshore wake simulations with the LES model PALM, pp 175-181.

Wu YT, Porté-Agel F (2012) Atmospheric turbulence effects on wind-turbine wakes: An LES study. Energies 5:5340-5362

Zhang W, Markfort CD, Porté-Agel F (2012) Near-wake flow structure downwind of a wind turbine in a turbulent boundary layer. Exp Fluids 52:12191235 

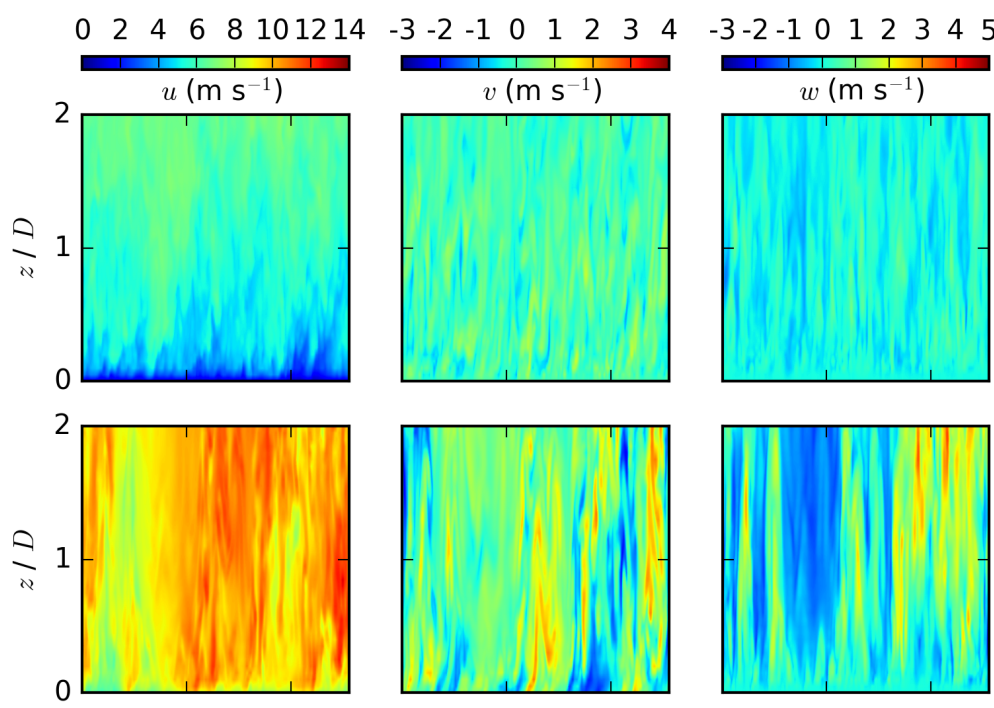

NBL
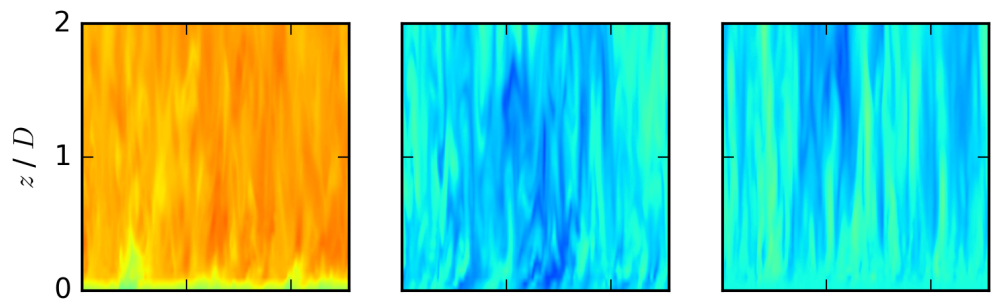

EBL
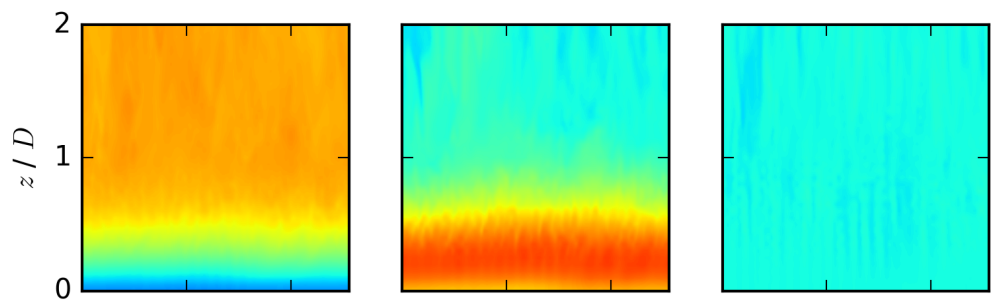

CBL
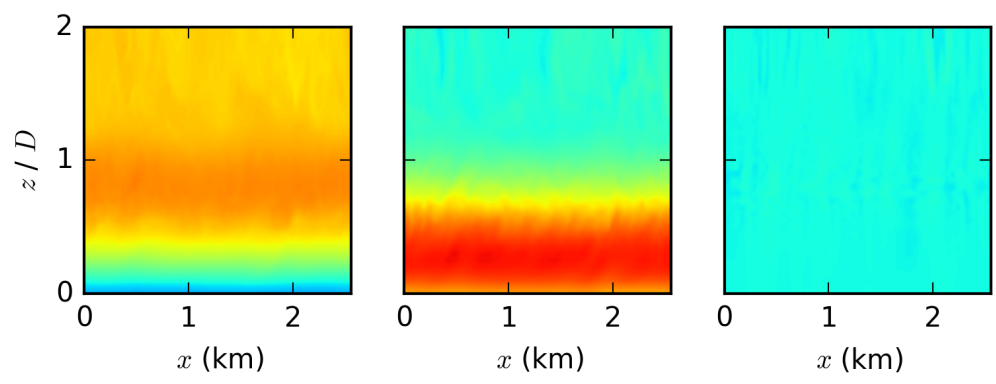

MBL

Fig. 11 Vertical cross-sections at $y_{0}$ of $u, v$, and $w$ for the neutral ABL equilibrium state (NBL, first row) of the precursor simulation in Englberger and Dörnbrack (2017a), the convective ABL state at $t=12 \mathrm{~h}$ (CBL, second row), the evening ABL state at $t=18 \mathrm{~h}$ (EBL, third row), the stable ABL state at $t=24 \mathrm{~h}$ (SBL, fourth row), and the morning ABL state at $t=29 \mathrm{~h}$ (MBL, fifth row) of the idealized diurnal-cycle simulation in Englberger and Dörnbrack (2017b). 

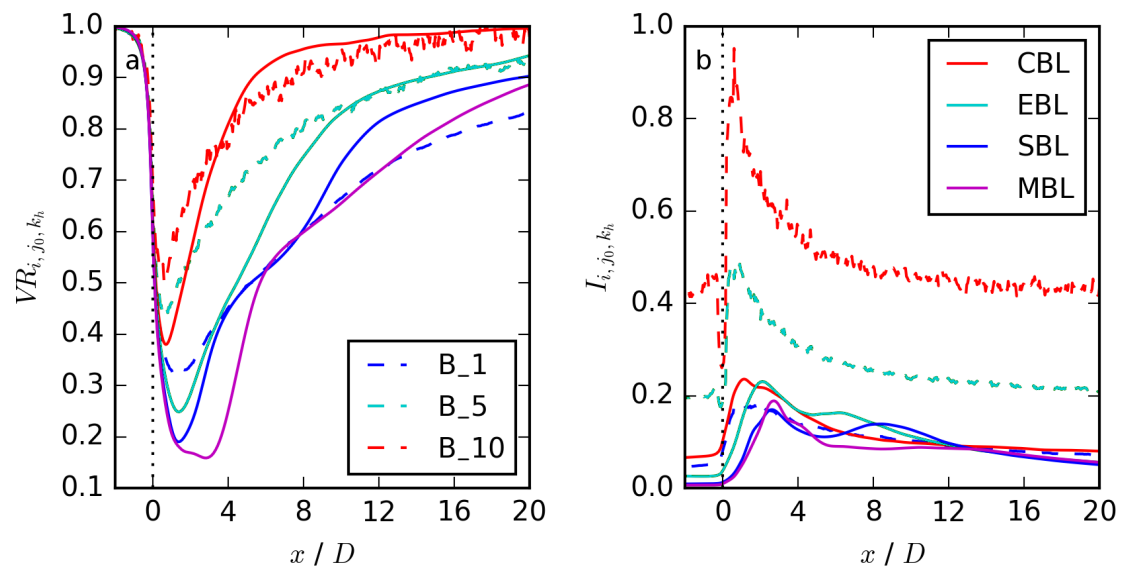

Fig. 12 A comparison of the streamwise dependency of $V R_{i, j_{0}, k_{h}}$ in $(a)$, and $I_{i, j_{0}, k_{h}}$ in $(b)$ between the wind-turbine simulations performed with the original version of the turbulence preserving model for $\alpha=1, \alpha=5$, and $\alpha=10$, and the convective ABL (CBL), the evening ABL (EBL), the stable ABL (SBL), and the morning ABL (MBL) situation resulting from the diurnal-cycle simulation of Englberger and Dörnbrack (2017b). 